\title{
Interactions between Behaviorally Relevant Rhythms and Synaptic Plasticity Alter Coding in the Piriform Cortex
}

\author{
Anne-Marie M. Oswald and Nathaniel N. Urban \\ Department of Biological Sciences, Center for the Neural Basis of Cognition, Carnegie Mellon University, Pittsburgh, Pennsylvania 15213
}

Understanding how neural and behavioral timescales interact to influence cortical activity and stimulus coding is an important issue in sensory neuroscience. In air-breathing animals, voluntary changes in respiratory frequency alter the temporal patterning olfactory input. In the olfactory bulb, these behavioral timescales are reflected in the temporal properties of mitral/tufted (M/T) cell spike trains. As the odor information contained in these spike trains is relayed from the bulb to the cortex, interactions between presynaptic spike timing and short-term synaptic plasticity dictate how stimulus features are represented in cortical spike trains. Here, we demonstrate how the timescales associated with respiratory frequency, spike timing, and short-term synaptic plasticity interact to shape cortical responses. Specifically, we quantified the timescales of short-term synaptic facilitation and depression at excitatory synapses between bulbar M/T cells and cortical neurons in slices of mouse olfactory cortex. We then used these results to generate simulated M/T population synaptic currents that were injected into real cortical neurons. M/T population inputs were modulated at frequencies consistent with passive respiration or active sniffing. We show how the differential recruitment of short-term plasticity at breathing versus sniffing frequencies alters cortical spike responses. For inputs at sniffing frequencies, cortical neurons linearly encoded increases in presynaptic firing rates with increased phase-locked, firing rates. In contrast, at passive breathing frequencies, cortical responses saturated with changes in presynaptic rate. Our results suggest that changes in respiratory behavior can gate the transfer of stimulus information between the olfactory bulb and cortex.

\section{Introduction}

Many air-breathing animals transition from slow passive respiration to fast active sniffing to investigate their environment (Youngentob et al., 1987; Thesen et al., 1993; Porter et al., 2007; Wesson et al., 2008b). Active sniffing improves odor detection, localization, and discrimination (Uchida and Mainen, 2003; Rajan et al., 2006; Kepecs et al., 2007; Frasnelli et al., 2009; Cury and Uchida, 2010), which suggests that odor information arriving during sniffing might be differentially processed in the olfactory pathway. Although sniffing alters activity patterns in both the olfactory bulb (Bathellier et al., 2008; Cury et al., 2010; Carey and Wachowiak, 2011, Shusterman et al., 2011) and piriform cortex (Kay, 2005; Mainland and Sobel, 2006), the influence of respiratory frequency on the transfer of odor information between these processing centers has not been investigated.

Respiration draws odorants across the nasal epithelium to activate olfactory receptor neurons (ORNs). In the olfactory bulb, ORN axons synapse with mitral and tufted cells $(\mathrm{M} / \mathrm{T})$, which, in

\footnotetext{
Received Dec. 18, 2011; revised Feb. 20, 2012; accepted March 11, 2012.

Author contributions: A.-M.M.O. and N.N.U. designed research; A.-M.M.O. performed research; A.-M.M.O. analyzed data; A.-M.M.O. and N.N.U. wrote the paper.

This work was supported by NIDCD Grant R03 DC011375 (A.-M.M.0.) and NIDCD Grants R01 DC0005798 and R01 DC011184 (N.N.U.). We thank T. Tzounopoulos for comments on early versions of this manuscript and B. Doiron for helpful discussions.

The authors declare no competing financial interests.

Correspondence should be addressed to Anne-Marie M. Oswald, Department of Neuroscience, University of Pittsburgh, A210 Langley Hall, Pittsburgh, PA 15260. E-mail: amoswald@pitt.edu.

DOI:10.1523/JNEUROSCI.6285-11.2012

Copyright $\odot 2012$ the authors $\quad 0270-6474 / 12 / 326092-13 \$ 15.00 / 0$
}

turn, project odor information to the piriform cortex. Odordriven responses in ORNs (Verhagen et al., 2007; Wesson et al., 2008a, 2009), M/T cells (Macrides and Chorover, 1972; Sobel and Tank, 1993; Kay and Laurent, 1999; Cang and Isaacson, 2003; Margrie and Schaefer, 2003), and cortical neurons phase lock to passive respiration cycles (Litaudon et al., 2003, 2008; Lei et al., 2006; Rennaker et al., 2007; Poo and Isaacson, 2009). M/T cells also phase lock to respiratory cycles at sniff frequencies (Bathellier et al., 2008; Cury and Uchida, 2010; Carey and Wachowiak, 2011, Shusterman et al., 2011). Furthermore, odor information can be encoded in the firing rate (FR) and/or latency of M/T cell spikes relative to the respiration cycle (Brody and Hopfield, 2003; Cang and Isaacson, 2003; Margrie and Schaefer, 2003; Bathellier et al., 2008; Cury and Uchida, 2010; Carey and Wachowiak, 2011).

Since passive breathing and active sniffing occur on different timescales, the transfer of odor information encoded in M/T spike trains may be influenced temporal properties of the synapses between the bulb and the cortex. Excitatory synapses between M/T cells and cortical neurons exhibit short-term facilitation and depression (Bower and Haberly, 1986; Hasselmo and Bower, 1990; Suzuki and Bekkers, 2006, 2011; Stokes and Isaacson, 2010) that could dynamically filter olfactory bulb input (Abbott and Regehr, 2004). Here, we demonstrate how afferent input delivered at different respiration frequencies engages shortterm plasticity at these synapses and affects cortical responses. We find that when simulated $\mathrm{M} / \mathrm{T}$ population inputs are modulated at sniffing frequencies, cortical neurons code increases in M/T firing rate with increasing phase-locked, firing rates. In contrast, 
when $\mathrm{M} / \mathrm{T}$ inputs are modulated at passive breathing frequencies, the recruitment of short-term synaptic depression results in cortical spike responses that saturate with changes in $\mathrm{M} / \mathrm{T}$ firing rate. Finally, we show that the expression of facilitation alters the gain of these cortical responses. These data suggest the transition from passive breathing to active sniffing in combination with shortterm plasticity shapes information transfer between the olfactory bulb and cortex.

\section{Materials and Methods}

Olfactory cortical slices were prepared from CBJ/BL6 mice of either sex, aged P11-P28. Only $15 \%$ of the recorded neurons were from mice $<\mathrm{P} 15$. Since recordings from these neurons did not differ significantly from the remainder of the population, it is unlikely that early development significantly affects our results. All surgical procedures followed the guidelines approved by the Carnegie Mellon Animal Welfare Committee. The mice were anesthetized with isoflurane and decapitated. The brain was exposed, removed from the skull, and immersed in ice-cold oxygenated $\left(95 \% \mathrm{O}_{2}-5 \% \mathrm{CO}_{2}\right.$ ) ACSF (in mm: $125 \mathrm{NaCl}, 2.5 \mathrm{KCl}, 25 \mathrm{NaHCO}_{3}, 1.25$ $\mathrm{NaH}_{2} \mathrm{PO}_{4}, 1.0 \mathrm{MgCl}_{2}, 25$ dextrose, $2 \mathrm{CaCl}_{2}$ ) (all chemicals from SigmaAldrich). Care was taken to ensure that the olfactory bulbs and lateral olfactory tract (LOT) remained intact. Coronal or horizontal slices (300 $\mu \mathrm{m}$ ) were made using a vibratome (Leica). The slices were maintained in $\mathrm{ACSF}$ at $37^{\circ} \mathrm{C}$ for $30 \mathrm{~min}$, and then rested at room temperature (20$22^{\circ} \mathrm{C}$ ) for at least $1 \mathrm{~h}$ before recording $\left(31-35^{\circ} \mathrm{C}\right)$.

Electrophysiology. Recordings were obtained from L2 principal neurons of piriform cortex. Neurons were visualized using infrared-differential interference contrast microscopy (Olympus). Pyramidal cells were typically identified by a primary apical dendrite that extended toward L1, while semilunar cells projected two to four apical dendrites to L1. Wholecell, current-clamp recordings of both pyramidal and semilunar cells were performed using a MultiClamp 700B amplifier (Molecular Devices). Data were low-pass filtered $(4 \mathrm{kHz})$ and digitized at $10 \mathrm{kHz}$ using an ITC-18 (InstruTECH) controlled by custom software written in IgorPro (Wavemetrics). Pipettes were pulled from borosilicate glass $(1.5 \mathrm{~mm}$, outer diameter) on a Flaming/Brown micropipette puller (Sutter Instrument) to a resistance of 6-12 M $\Omega$. The intracellular solution consisted of the following (in mM): $130 \mathrm{~K}$-gluconate, $5 \mathrm{KCl}, 2 \mathrm{MgCl}_{2}, 4$ ATP-Mg, 0.3 GTP, 10 HEPES, and 10 phosphocreatine.

The intrinsic properties of the neurons were assessed using a series of hyperpolarizing and depolarizing current steps ( -50 to $800 \mathrm{pA}, 1 \mathrm{~s} \mathrm{du}-$ ration). At the onset of a depolarizing step current, pyramidal cells typically fire a high-frequency spike doublet or burst after which firing rates adapted, whereas semilunar cells are regular spiking (Suzuki and Bekkers, 2006). The spike rate adaptation ratio was taken as the last interspike interval (ISI) divided by the first ISI. Bursting pyramidal neurons had adaptation ratios that were significantly greater $(15.5 \pm 1.5)$ than regular spiking neurons $(1.5 \pm 0.1 ; p<0.01)$. Moreover, pyramidal neurons had significantly lower membrane time constants $\left(\tau_{\mathrm{m}}, 16.7 \pm\right.$ $1.5 \mathrm{~ms})$ and input resistances $\left(R_{\mathrm{n}}, 128 \pm 11 \mathrm{M} \Omega\right)$ than regular spiking, semilunar neurons $\left(\tau_{\mathrm{m}}, 30.1 \pm 3.7 \mathrm{~ms} ; R_{\mathrm{n}}, 244 \pm 22 \mathrm{M} \Omega\right.$; $\left.p<0.01\right)$. The anatomical and electrophysiological properties of the recorded neurons were consistent with previous descriptions of bursting pyramidal cells and regular spiking semilunar cells (Suzuki and Bekkers, 2006, 2011).

LOT stimulation. To assess synaptic inputs from the olfactory bulb to cortex, the LOT in Lla of the piriform cortex was stimulated with single pulses (300-1000 $\mu \mathrm{A} ; 100 \mu$ s pulse duration) using either monopolar or bipolar, glass microelectrodes. Stimulus intensity was chosen as the lowest current that evoked EPSPs on at least $80 \%$ of stimulus trials (single pulses, $5 \mathrm{~s}$ between trials). Importantly, the amplitudes of these EPSPs (0.31-10.8 mV) were comparable with values recorded at threshold stimulation intensity $(0.23-11.4 \mathrm{mV})$, suggesting an increase in the reliability of fiber activation rather than the recruitment of additional fibers. The reported EPSP amplitudes were measured at resting membrane potentials $(-63$ to $-67 \mathrm{mV})$. To ensure that the recorded PSPs were predominantly excitatory, neurons were depolarized to $-45 \mathrm{mV}$ during LOT stimulation. If the PSPs reversed or showed a substantial inhibitory com-
Table 1. Synaptic parameters

\begin{tabular}{|c|c|c|c|c|c|c|c|c|}
\hline \multicolumn{3}{|c|}{$\tau_{\text {rec }}(\mathrm{ms})$} & \multicolumn{2}{|l|}{$\tau_{\mathrm{fac}}(\mathrm{ms})$} & \multicolumn{2}{|l|}{$E$} & \multicolumn{2}{|l|}{ U } \\
\hline & Fit & Model & Fit & Model & Fit & Model & Fit & Model \\
\hline & 5 & 85 & $1171 \pm$ & 1200 & $3.0=$ & 3 & 0.36 & 0.35 \\
\hline FD & $163 \pm 25$ & 160 & $910 \pm 38$ & 900 & $2.6 \pm 0.2$ & 3 & $0.24 \pm 0.02$ & 0.25 \\
\hline D & $148 \pm 18$ & 150 & n/a & $n / a$ & $4.5 \pm 0.3$ & 5 & $0.80 \pm 0.02$ & 0.8 \\
\hline
\end{tabular}

The mean \pm SE synaptic parameters obtained from fits (left columns) to recorded cortical responses during lateral olfactory tract stimulation for facilitation-dominant (F), facilitating-depressing (FD), and depression-dominant (D) synapses. The corresponding values used to model $\mathrm{M} / \mathrm{T}$ population synaptic currents are also listed (right columns). $E$, Total synaptic efficacy; $\tau_{\text {fac }}$, time constant for facilitation; $\tau_{\text {rec }}$, time constant for recovery from depression; and $U$, the utilization of efficacy on a single pulse at rest (fully recovered and nonfacilitated).

ponent, these neurons were excluded from analysis. Nonetheless, since we did not block inhibitory synaptic transmission, we cannot entirely rule out a contribution of inhibition to synaptic amplitudes. EPSP amplitude (in millivolts) was taken as the difference between the peak of the EPSP and the membrane potential at EPSP onset. We also obtained the time constant for the decay $\left(\tau_{\alpha}\right)$ of the EPSP by fitting the normalized (peak-baseline $=1)$ EPSP amplitude $\left(A_{t}\right.$, where $t$ is time in milliseconds) with an $\alpha$ function (Eq. 1) as follows:

$$
A_{t}=\frac{t}{\tau_{\alpha}} e^{\left((1-t) / \tau_{\alpha}\right)}
$$

Analysis of short-term plasticity. To study short-term synaptic plasticity at excitatory synapses, trains ( $7 \mathrm{~s}$ duration) of Poisson distributed stimulus pulses (mean rate, $10 \mathrm{~Hz}$; $\sim 50$ pulses) were delivered to the LOT. The shortest interpulse interval (IPI) within the train was $10 \mathrm{~ms}$ to ensure that PSP amplitudes could be resolved and measured without contamination by stimulus artifacts. The change in synaptic amplitude [relative amplitude (RA)] was measured as the amplitude of each EPSP $\left(A_{i}\right)$ relative to the first EPSP of the train $\left(A_{o}\right)$ as follows:

$$
\mathrm{RA}=A_{i} / A_{0} .
$$

RA values $<1$ were indicative of short-term depression, while values $>1$ indicated facilitation. To obtain the time constants for facilitation and depression, we fit the changes in RA over the Poisson stimulus train using a phenomenological model of short-term plasticity adapted from the study by Markram et al. (1998). The premise of the model is that, on any given stimulus pulse $(n)$, the RA is the product of the maximum strength or efficacy $(E)$ of a synapse, the proportion of synaptic efficacy that is used $(u)$ and becomes immediately unavailable, and the proportion of efficacy that remains $(r)$ as follows:

$$
R A_{n}=E \cdot r_{n} \cdot u_{n} .
$$

For facilitating synapses, the proportion that is used $\left(u_{n}\right)$ varies depending on the time between stimulus pulses (IPI) (in milliseconds) and the time constant for facilitation $\left(\tau_{\mathrm{fac}}\right)$ as follows:

$$
u_{n+1}=u_{n} e^{\left(-\mathrm{IPI} / \tau_{\mathrm{fac}}\right)}+U\left(1-u_{n} e^{\left(-\mathrm{IPI} / \tau_{\mathrm{fac}}\right)}\right) .
$$

For all synapses, $U$ is the utilization of efficacy on a single pulse at rest. For solely depressing synapses, $u_{n}=U$ and is constant. Once used, $u_{n}$ becomes immediately unavailable for the next pulse and the remaining efficacy $\left(r_{n}\right)$ is decremented (or depressed) by $u_{n}$. The remainder recovers from this depression with time constant $\left(\tau_{\text {rec }}\right)$ according to the following:

$$
\left.r_{n+1}=r_{n}\left(1-u_{n+1}\right) e^{\left(-\mathrm{IPI} / \tau_{\mathrm{rec}}\right)}+1-e^{\left(-\mathrm{IPI} / \tau_{\mathrm{rec}}\right)}\right) .
$$

For each neuron, the model was fit using an iterative procedure that minimized the root-mean-squared error (RMSE) between the recorded RA and the predicted amplitudes based on the model. During fitting, $E$, $\tau_{\text {fac }}, \tau_{\text {rec }}$, and $U$ were free parameters. Solely depressing synapses were fit using Equations 3 and 5 and three parameters: $E, \tau_{\text {rec }}$, and $U$. Facilitating synapses were fit using Equations 3-5 and four parameters: $E, \tau_{\mathrm{fac}}, \tau_{\mathrm{rec}}$ and $U$. All fit and RMSE values were consistent with values reported by Markram et al. (1998) for other types of excitatory cortical synapses. In later sections of the paper, $E, \tau_{\mathrm{fac}}, \tau_{\text {rec }}$, and $U$ were fixed according to their mean values (Table 1) for simulations of population currents. 
Simulated olfactory bulb population input to cortical neurons. Based on the results of our plasticity experiments, we generated stimuli that simulated the drive from the olfactory bulb to cortical neurons during passive respiration $(2 \mathrm{~Hz})$ or active sniffing $(8 \mathrm{~Hz})$. We modeled these inputs as the summed excitatory synaptic current from a population of 20 $\mathrm{M} / \mathrm{T}$ cells. To create these stimuli, individual $\mathrm{M} / \mathrm{T}$ cells were represented by Poisson distributed spike trains (50 s duration) with FRs that were modulated at $2 \mathrm{~Hz}$ (passive respiration) or $8 \mathrm{~Hz}$ (active sniffing). Then we convolved these spike trains with simulated synaptic currents that were scaled by short-term plasticity. Finally, all individual simulated $\mathrm{M} / \mathrm{T}$ current inputs were summed to create a population current that was injected to cortical principal neurons. Hereafter in the text, frequencies (in hertz) related to spike activity are denoted as FR, while those related to respiration are referred to as modulation frequencies or rhythms.

Sinusoidally modulated M/T spike trains. For simplicity, we initially modeled the changes in $\mathrm{M} / \mathrm{T}$ activity during respiration by sinusoidally modulating the firing rate of the Poissondistributed spike trains at 2 or $8 \mathrm{~Hz}$. To mimic odor-evoked increases in M/T cell activity, the amplitude of the sinusoidal modulation (FR) was set at 2, 6, 10, or $14 \mathrm{~Hz}$. This sinusoidally modulated firing rate was added to a baseline firing rate that was randomly drawn from a Gaussian distribution with mean of $10 \mathrm{~Hz}$ and $\mathrm{SD}$ of $5 \mathrm{~Hz}$. When averaged across cycles and the population, the resulting peak firing rates ranged from 12 to $24 \mathrm{~Hz}$. However, within a cycle, the instantaneous firing rates (1/interspike interval) of individual $\mathrm{M} / \mathrm{T}$ spike trains were much higher $(60-100 \mathrm{~Hz})$. These firing rates are consistent with the mean changes in firing rate recorded in awake animals in vivo (Rinberg et al., 2006; Davison and Katz, 2007; Fuentes et al., 2008; Cury and Uchida, 2010).

Burst spike trains. We also created a set of stimuli that mimicked the burst-like responses of $\mathrm{M} / \mathrm{T}$ cells recorded in vivo. To generate these stimuli, a gallery of M/T FR patterns (see Fig. 8) was modeled based on cycle peristimulus histograms (PSTHs) recorded during passive respiration (Carey and Wachowiak, 2011) or active sniffing (Cury and Uchida, 2010). For each simulated $\mathrm{M} / \mathrm{T}$ cell in the population $(n=$ 20 ), an FR pattern was randomly selected from the gallery and scaled to a peak firing rate drawn from a Gaussian distribution with a mean of 150,200 , or $250 \mathrm{~Hz}(\mathrm{SD} \pm 50 \mathrm{~Hz})$. In addition, the onset of the FR pattern relative to the cycle was jittered according to a Gaussian distribution with mean of $10 \mathrm{~ms}$ ( $\mathrm{SD}, \pm 5 \mathrm{~ms}$ ). Then the FR pattern was repeated at $500 \mathrm{~ms}$ (2 $\mathrm{Hz})$ or $125 \mathrm{~ms}(8 \mathrm{~Hz})$ intervals for $50 \mathrm{~s}$. Finally, these repetitive FR patterns were used to generate Poisson distributed spike times.

Simulated synaptic currents. To create population currents to inject to cortical neurons, each sinusoidal or burst modulated $\mathrm{M} / \mathrm{T}$ spike train was convolved with $\alpha$ function synaptic currents (Eq. $1 ; \tau_{\alpha}, 10 \mathrm{~ms}$ ). The initial amplitudes of the $\alpha$ functions, were either $20 \mathrm{pA}$ for facilitating synapses or $40 \mathrm{pA}$ for depressing synapses. The amplitudes and time constant of
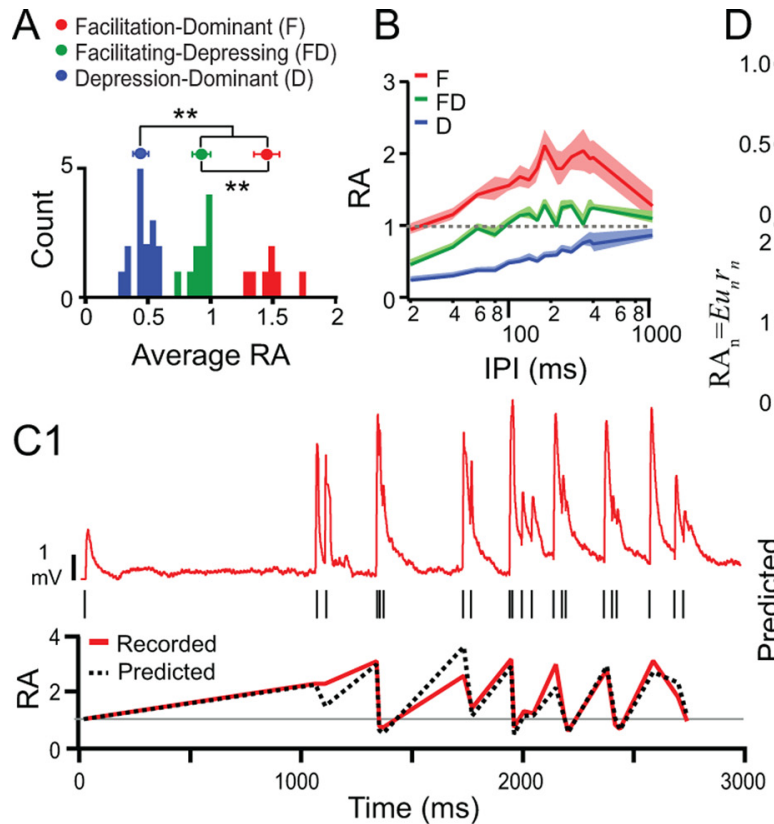

D Spikes
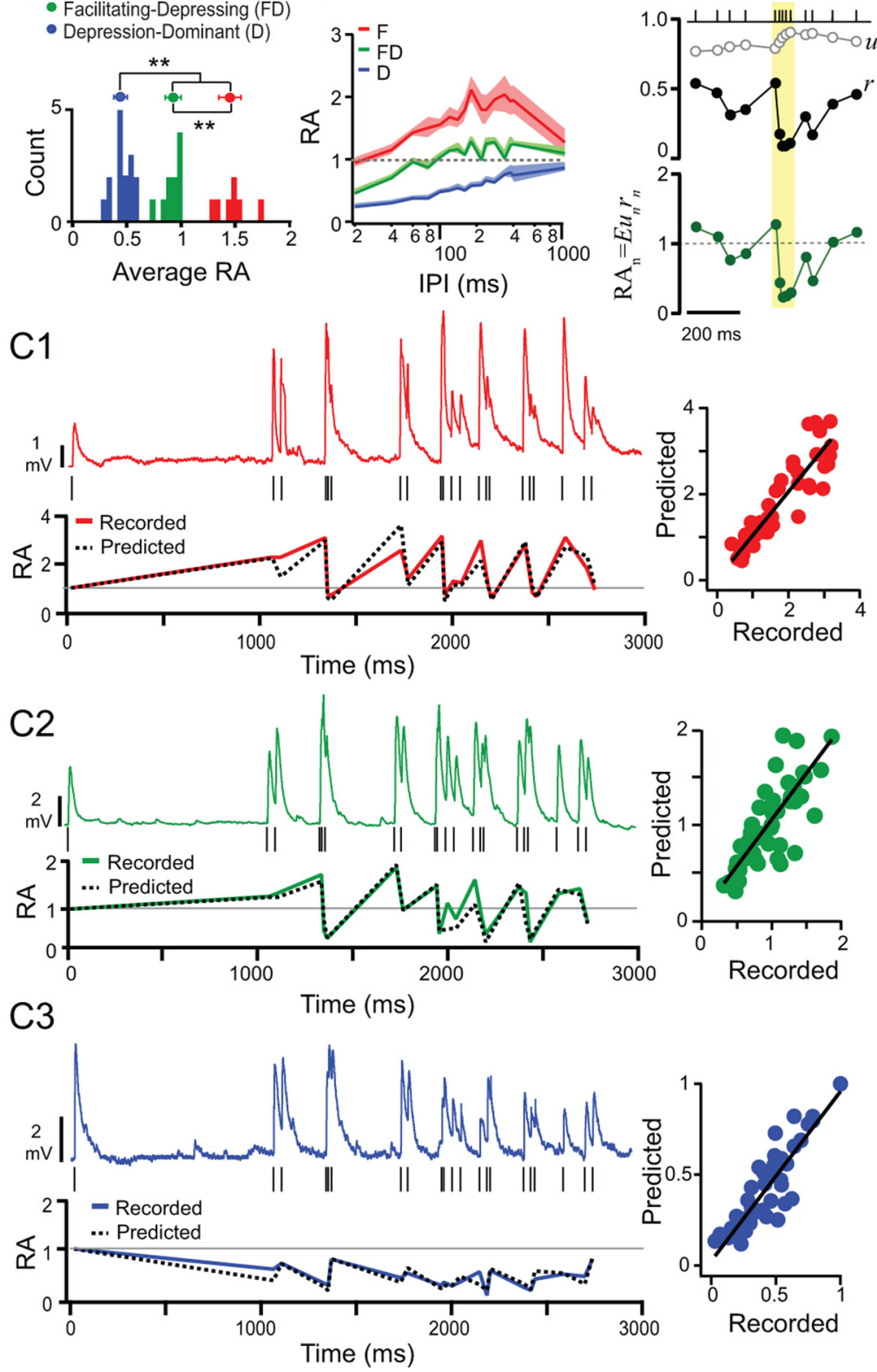

Figure 1. Short-term plasticity at LOT synapses. $\boldsymbol{A}$, Histogram of the average RA of EPSPs over the entire stimulus train. The distribution is trimodal showing facilitation-dominant (F) synapses (red bars), facilitating-depressing (FD) synapses (green bars), and depression-dominant (D) synapses (blue bars). The mean RAs (circles) for each synapse type were significantly different $\left({ }^{* *} p<0.01\right.$, unpaired $t$ test). $\boldsymbol{B}$, The mean RA ( \pm SE) for a given IPI is plotted for each synapse type ( $F$, red; FD, green; $D$, blue). $\boldsymbol{C}$, Top traces, Example recordings of EPSPs (colored) from cortical neurons in response to Poisson stimulation of the LOT (black pulses). Bottom plot, The recorded RA (solid colored lines) and predicted RA based on the model (dashed black line) for each stimulus pulse plotted against time. Right, The linear relationship between the recorded and predicted RA. Model fits to the data minimized the RMSE between the recorded and predicted RA for each EPSP. C1, Facilitation-dominant (F) synaptic input (red): RMSE, 0.16; linear fit: slope, 1.0; $R$, 0.9. C2, Facilitating-depressing (FD) synaptic input (green): RMSE, 0.10; linear fit: slope, 0.96; $R, 0.75$. C3, Depression-dominant (D) synaptic input (blue): RMSE, 0.01; linear fit: slope, $1.0 ; R, 0.85$. D. The change in utilization (u) (gray circles) and the remainder ( $r$ ) (black circles) parameters of the model with presynaptic spike times (top trace). Increases in spike rate (yellow box) moderately increase $u$ but dramatically decrease $r$. This results in a substantial decrease in predicted RA according to $R A=E \cdot u \cdot r$ (green circles).

the $\alpha$ functions were chosen such that current injection of a single $\alpha$ function at the soma produced a simulated EPSP that was $\sim 2-6 \mathrm{mV}$ and decayed to baseline within 50-100 ms of onset depending on the input resistance and time constant of the recorded neuron (data not shown).

Next, the amplitude of each $\alpha$ function synapse was scaled based on the preceding IPI according to Equations $3-5$ and mean values of $E, \tau_{\mathrm{fac}}, \tau_{\mathrm{rec}}$, and $U$ obtained for fits to the short-term synaptic plasticity data (Table 
A1
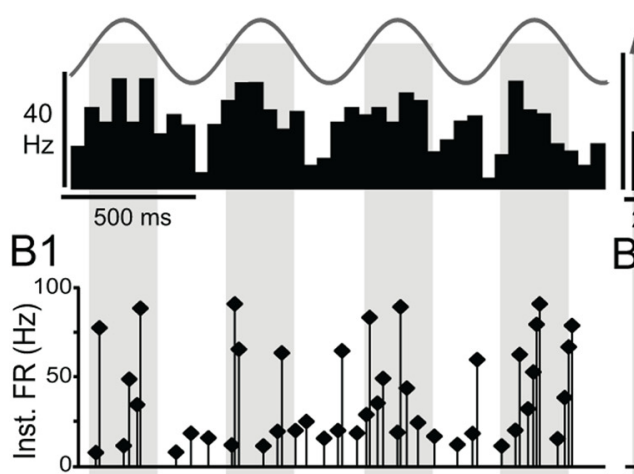

C1
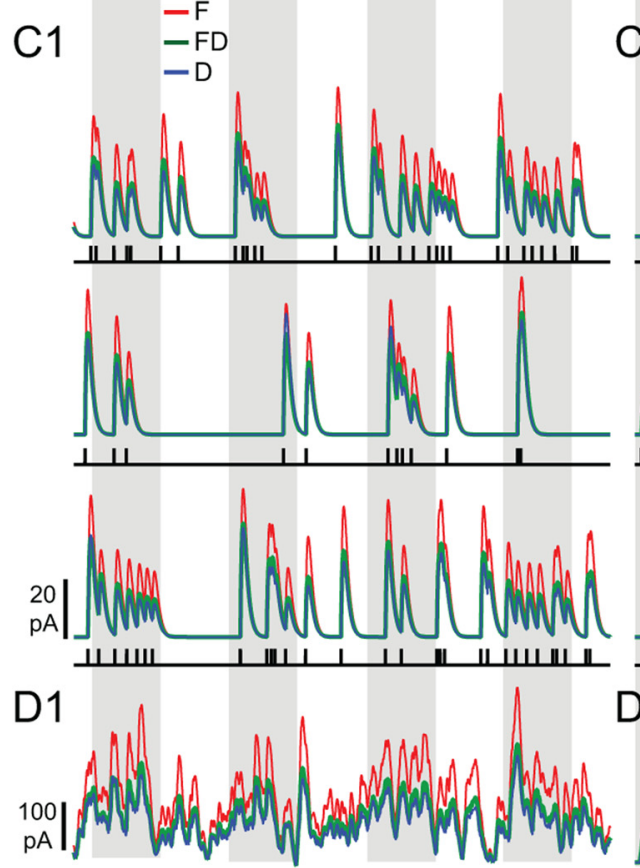

E

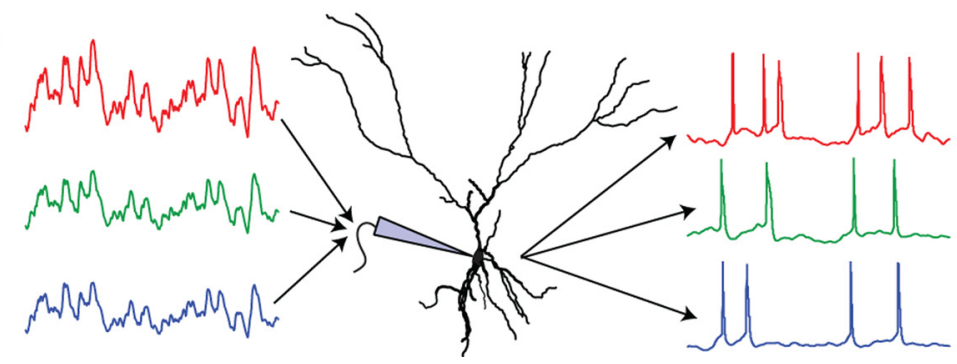

Figure 2. Simulated $M / T$ population currents. $\boldsymbol{A}$, Histogram of the average spike rate of the simulated M/T population (black bars) over a number of cycles for $2 \mathrm{~Hz}(\boldsymbol{A} 1)$ or $8 \mathrm{~Hz}$ (A2) sinusoidal modulations (gray). $\boldsymbol{B}$, The instantaneous firing rate (1/ISI) of a single simulated $\mathrm{M} / \mathrm{T}$ cell at $2 \mathrm{~Hz}(\boldsymbol{B} 1)$ or $8 \mathrm{~Hz}$ (B2). C, Examples of individual M/T simulated current inputs during $2 \mathrm{~Hz}(\boldsymbol{C}$ ) or $8 \mathrm{~Hz}$ (C2) modulation. Poisson distributed spike times (black pulses) were replaced with $\alpha$ function currents that were scaled based on the timescales of facilitation-dominant (F) (red), facilitating-depressing (FD) (green), or depression-dominant (D) (blue) synaptic responses. $\boldsymbol{D}$, Twenty $\alpha$ function sequences, comparable with those shown in $\boldsymbol{C}$, were summed to form excitatory M/T population currents (colors as in $\boldsymbol{C}$ ) that were modulated at $2 \mathrm{~Hz}(\boldsymbol{D} 1)$ or $8 \mathrm{~Hz}(\boldsymbol{D 2})$. $\boldsymbol{E}$, These stimuli (left) were then directly injected at the somas of cortical neurons to drive spike responses (right; colors as in $\mathbf{C}$ ). between $\mathrm{M} / \mathrm{T}$ cells and cortical neurons. An additional 18 burst-like stimuli were created to account for the two simulated respiratory rhythms ( 2 and $8 \mathrm{~Hz}$ ), the three different M/T peak firing rates $(150,200,250 \mathrm{~Hz})$, and the three types of synaptic plasticity recorded at excitatory synapses between $\mathrm{M} / \mathrm{T}$ cells and cortical neurons. For the majority of cortical neurons, the stimulus current amplitudes were sufficiently suprathreshold. However, in some neurons, a subthreshold bias current $(20-50$ $\mathrm{pA})$ was applied to ensure adequate firing rates (minimum, $4 \mathrm{~Hz}$ ) for analysis. In cases in which a bias current was added, it was added uniformly across all stimuli tested.

It should be noted that the individual, simulated M/T spike trains are correlated solely through common modulation at respiratory frequencies. Correlated activity among M/T cells due to circuit interactions in the olfactory bulb (Urban and Sakmann, 2002; Galan et al., 2006; Giridhar et al., 2011) is not modeled, but would be expected to enhance our results.

Analysis of cortical spike responses and statistics. The mean cortical firing rates in response to the simulated $\mathrm{M} / \mathrm{T}$ population currents were calculated as the total number of spikes divided by the $50 \mathrm{~s}$ stimulus duration. The phasic responses of cortical neurons were quantified using cycle histograms. The cycle lengths were $500 \mathrm{~ms}(2 \mathrm{~Hz})$ and $125 \mathrm{~ms}(8 \mathrm{~Hz})$, and each cycle was divided 10 bins (50 and $12.5 \mathrm{~ms} \mathrm{du}$ ration, respectively). The number spikes per bin were summed over all cycles. Firing rate cycle histograms were calculated by dividing the average number spikes per bin (over all cycles) by the bin duration. All statistics are reported as mean $\pm \mathrm{SE}$, and significance was assessed using Student's paired and unpaired $t$ tests.

\section{Results}

We investigated how respiratory rhythms and the recruitment of short-term synaptic plasticity at bulb-to-cortex synapses affect the transfer of olfactory bulb population activity to the cortex. We initially characterized the synaptic responses from principal neurons $(n=32)$ in L2 of anterior olfactory cortex $(n=6)$ and anterior piriform cortex $(n=26)$ during LOT stimulation. Based on our results, we created simulated $\mathrm{M} / \mathrm{T}$ population currents that were used to drive spiking in cortical principal neurons $(n=19)$. We then explored how the interactions between the timescales of short-term plasticity at M/T synapses and respiratory rhythms give rise to different cortical responses.
1). Finally, the 20 individual, simulated $M / T$ synaptic current sequences were summed to produce a population stimulus current (50 s duration) that was directly injected at the somas of cortical neurons.

Together, there were 24 sinusoidally modulated, M/T population current stimuli created to account for the two simulated respiratory rhythms ( 2 and $8 \mathrm{~Hz}$ ), the four different average firing rates $(12,16,20,24 \mathrm{~Hz}$ ), and the three types of synaptic plasticity recorded at excitatory synapses

\section{Short-term synaptic plasticity at LOT synapses}

The transfer of odor information represented by M/T spike trains is likely influenced by the short-term plasticity at synapses between $\mathrm{M} / \mathrm{T}$ cells and cortical neurons in the LOT. To characterize plasticity at these synapses, we stimulated the LOT (layer 1a) with a train of Poisson distributed pulses (7 s duration; mean rate, 10 
$\mathrm{Hz}$ ). This stimulus is advantageous because it allows the quantification of short-term plasticity over a range of stimulus frequencies $(1-100 \mathrm{~Hz})$ using a variety of IPIs between 10 and $1000 \mathrm{~ms}$. These IPIs are consistent with interspike intervals found in $\mathrm{M} / \mathrm{T}$ spike trains that have instantaneous firing rates ranging from 1 to $200 \mathrm{~Hz}$ (Cury et al., 2010; Carey and Wachowiak, 2011; Shusterman et al., 2011). We measured short-term plasticity as the ratio of the amplitude of each EPSP of the train relative to the first EPSP of the train (RA). Changes in RA that were $>1$ indicated short-term facilitation, while RA values $<1$ indicated depression. When we assessed the average RA across the train, we found that the distribution of synapse types was strikingly trimodal (Fig. $1 A)$. This was surprising because previous reports have described these inputs dichotomously as either facilitating or depressing (Bower and Haberly, 1986; Hasselmo and Bower, 1990; Suzuki and Bekkers, 2006, 2011). We found facilitation-dominant (F) synapses $(n=7$; red) that had an average RA of $1.45 \pm 0.06$ (Fig. $1 A$ ) and values near 1 for only the shortest IPIs (Fig. $1 B$ ). Facilitating-depressing (FD) synapses $(n=10$; green) had an average RA of $0.93 \pm 0.03$ and RA values $<1$ for short IPIs and RA $>1$ for longer IPIs. Depression-dominant (D) synapses $(n=15$; blue) had an average RA of $0.44 \pm 0.02$ and rarely had RA values $>1$ for any IPI. Example traces recorded from neurons receiving each type of synapse are shown in Figure 1C1-C3.

The EPSPs of depression-dominant synapses had significantly greater initial amplitudes $(\mathrm{D}, 7.8 \pm 4.4 \mathrm{mV})$ than synapses that showed facilitation (F and FD, $2.4 \pm 2.3 \mathrm{mV} ; p<0.01$ ), but the synaptic decays did not differ $\left(\tau_{\alpha}\right.$ : facilitating, $14.3 \pm 1.3 \mathrm{~ms}$; depressing, $14.5 \pm 1.4 \mathrm{~ms} ; p=0.90$ ) (see Eq. 1, Materials and Methods). The majority of neurons that received solely depressing input $(n=15)$ were regular spiking (adaptation ratio, $\leq 1)$ (see Materials and Methods) consistent with semilunar cells. Alternatively, most neurons that received facilitating inputs $(n=$ 17) were bursting neurons (adaptation ratio, $>4$ ) suggestive of pyramidal cells. Since these results are consistent with previous characterizations of synaptic inputs to principal neurons in piriform cortex, we focus the remainder of the study on the general role of short-term plasticity in information coding rather than the specific differences between pyramidal and semilunar cells.

To obtain the timescales of plasticity for each type of synapse, we fit the relationship between the RA of a given EPSP in the train and the preceding IPI using a phenomenological model for short-term plasticity (Markram et al., 1998) (see Materials and Methods). The premise of the model is that, on any given stimulus pulse, the relative synaptic strength (RA) is the product of the maximum efficacy $(E)$ of a synapse, the proportion of efficacy used $(u)$ on the current pulse that becomes immediately unavailable for the next pulse, and the proportion that remains available $(r)$ (Fig. $1 D$, green circles). For facilitating synapses (F, FD), $u$ is incremented on each pulse and decays to its initial value $(U)$ according to the time constant for facilitation, $\tau_{\text {fac }}$ (Eq. 4; Fig. $1 D$, open circles). For solely depressing synapses (D), $u=U$ and the proportion used on each pulse is constant. For both facilitating and depressing synapses, $r$ is decremented by $u$ and recovers between pulses according to the time constant $\tau_{\text {rec }}$ (Eq. 5 ; Fig. $1 D$, black circles). The values of $E, U, \tau_{\text {fac }}$, and $\tau_{\text {rec }}$ were obtained from model fits to each data set and the means are reported for each synapse type (F, FD, and D) in Table 1. Facilitating synapses (F, FD) were described by two time constants $\left(\tau_{\text {fac }}\right.$ and $\left.\tau_{\text {rec }}\right)$, while solely depressing (D) synapses are described by just one, $\tau_{\text {rec }}$. Thus, all synapses (F, FD, D) recover from short-term depression described by $\tau_{\text {rec }}$, but only a subset of synapses (F, FD) express facilitation described by $\tau_{\text {fac }}$.
Table 2. Cortical firing rates in response to sinusoidally modulated, simulated $M / T$ population currents: mean cortical firing rate

\begin{tabular}{|c|c|c|c|c|c|c|}
\hline & \multicolumn{6}{|c|}{ Simulated respiratory rhythm } \\
\hline & \multicolumn{3}{|l|}{$2 \mathrm{~Hz}$ rhythm } & \multicolumn{3}{|l|}{$8 \mathrm{~Hz}$ rhythm } \\
\hline & \multicolumn{6}{|l|}{ Plasticity } \\
\hline & $\mathrm{F}$ & FD & $D$ & $\mathrm{~F}$ & $\mathrm{FD}$ & D \\
\hline \multicolumn{7}{|l|}{$\mathrm{M} / \mathrm{T} F \mathrm{~F}(\mathrm{~Hz})$} \\
\hline 12 & $6.3 \pm 1.1$ & $5.3 \pm 1.0$ & $3.8 \pm 0.7$ & $6.9 \pm 1.3$ & $5.3 \pm 0.8$ & $3.6 \pm 0.5$ \\
\hline 16 & $9.0 \pm 1.0$ & $6.1 \pm 0.9$ & $4.9 \pm 0.7$ & $10.4 \pm 1.5$ & $6.4 \pm 0.9$ & $5.3 \pm 0.7$ \\
\hline 20 & $10.7 \pm 1.2$ & $6.5 \pm 0.8$ & $5.3 \pm 0.7$ & $11.4 \pm 1.7$ & $7.5 \pm 1.0$ & $5.7 \pm 0.5$ \\
\hline 24 & $12.3 \pm 1.5^{\dagger t}$ & $7.5 \pm 0.9^{t \dagger}$ & $6.0 \pm 0.7^{\dagger \dagger}$ & $12.7 \pm 1.7^{\dagger \dagger}$ & $8.5 \pm 1.0^{t+}$ & $6.8 \pm 0.8^{t \dagger}$ \\
\hline Range $(\mathrm{Hz})$ & $6.1 \pm 0.5^{\mathrm{tt}}$ & $2.9 \pm 0.8$ & $2.2 \pm 0.3$ & $5.8 \pm 0.6^{t \dagger}$ & $3.4 \pm 0.4$ & $3.0 \pm 0.5$ \\
\hline
\end{tabular}

Mean firing rates $(\mathrm{Hz})$ of cortical neurons in response to $\mathrm{M} / \mathrm{T}$ population current stimuli. These rates are shown for all combinations of simulated respiration frequency ( 2 or $8 \mathrm{~Hz}$ rhythms), presynaptic $M / T$ firing rate ( $F R, 12-24 \mathrm{~Hz}$ ), and synaptic plasticity ( $F$, facilitation dominant; $F D$, facilitating-depressing; $D$, depression dominant).

Mean cortical firing rates increased significantly for comparisons between presynaptic rates of 12 and $24 \mathrm{~Hz}\left({ }^{4 \dagger} p<\right.$ 0.01 , paired $t$ test) but not for discrete comparisons (i.e., $12 \mathrm{vs} 16,16 \mathrm{vs} 20,20 \mathrm{vs} 24$ ). Furthermore, the range of mean cortical firing rates that represent the $12-24 \mathrm{~Hz}$ change in presynaptic rate was significantly greater for $\mathrm{F}$ synapses $\left({ }^{+\dagger} p<0.01\right.$, paired $t$ test) than the $\mathrm{FD}$ or D synapses.

For the synaptic responses shown in Figure $1 C 1-C 3$, the predicted change in RA based on the model is plotted versus time. The fitting procedure minimized the RMSE between the predicted (dashed line) and recorded (solid colored lines) RA for a given sequence of stimulus pulses. Overall, the synaptic responses were well fit by the model, as the average RMSE for facilitating synapses was $0.12 \pm 0.02$, and for depressing synapses, $0.02 \pm$ 0.005 . The relationship between the predicted and recorded amplitudes (Fig. 1C1-C3, right) was linear, which also indicates a good fit between model and data (facilitating synapses: $R, 0.7 \pm$ 0.03 ; depressing synapses: $R, 0.8 \pm 0.03$ ). F synapses had a significantly shorter time constant for depression $\left(\tau_{\text {rec }}, 86 \pm 16 \mathrm{~ms}\right)$ than FD $(163 \pm 26 \mathrm{~ms})$ or D $(147 \pm 18 \mathrm{~ms} ; p<0.05)$ synapses. F synapses also had a significantly longer time constant for facilitation $\left(\tau_{\mathrm{fac}}, 1171 \pm 94 \mathrm{~ms}\right)$ than FD synapses $(910 \pm 38 \mathrm{~ms} ; p<$ $0.05)$. In the following sections, we use this model to explore how short-term synaptic plasticity in a simulated population of $\mathrm{M} / \mathrm{T}$ inputs influences information transfer between the olfactory bulb and the cortex.

\section{Simulated population input from the olfactory bulb}

To investigate how synaptic dynamics influence the responses of cortical neurons to olfactory bulb inputs, we used the short-term plasticity model to simulate the synaptic current from a presynaptic population of $20 \mathrm{M} / \mathrm{T}$ cells. This current was then directly injected to real pyramidal or semilunar cortical neurons. The main advantage of driving cortical responses with simulated population currents is that each $\mathrm{M} / \mathrm{T}$ spike train is simulated independently of the other population inputs. This better represents the response heterogeneity of the $\mathrm{M} / \mathrm{T}$ population (Padmanabhan and Urban, 2010). The spike trains of individual M/T cells were simulated using time-varying Poisson processes with baseline spike rates randomly chosen from a Gaussian distribution (mean rate of $10 \mathrm{~Hz}$ and $\mathrm{SD}$ of $5 \mathrm{~Hz}$ ). To simulate odor-evoked increases in firing rate, $2,6,10$, or $14 \mathrm{~Hz}$ was added to this baseline rate. To mimic firing patterns during respiration, these increases in firing rate were sinusoidally modulated at $2 \mathrm{~Hz}$ (passive breathing) or $8 \mathrm{~Hz}$ (active sniffing). Together, this resulted in simulated presynaptic $\mathrm{M} / \mathrm{T}$ firing rates that, when averaged across cycles and the population, were 12, 16, 20, or $24 \mathrm{~Hz}$. In Figure $2 A$, we show the rhythmicity of the simulated spike rate of the M/T population over a number of 2 or $8 \mathrm{~Hz}$ cycles. Although average spike rates ranged between 12 and $24 \mathrm{~Hz}$, the instanta- 
Table 3. Cortical firing rates in response to sinusoidally modulated, simulated $\mathrm{M} / \mathrm{T}$ population currents: peak cortical firing rate

\begin{tabular}{|c|c|c|c|c|c|c|}
\hline & \multicolumn{6}{|c|}{ Simulated respiratory rhythm } \\
\hline & \multicolumn{3}{|l|}{$2 \mathrm{~Hz}$ rhythm } & \multicolumn{3}{|l|}{$8 \mathrm{~Hz}$ rhythm } \\
\hline & \multicolumn{6}{|l|}{ Plasticity } \\
\hline & $\mathrm{F}$ & FD & D & $\mathrm{F}$ & $\mathrm{FD}$ & D \\
\hline \multicolumn{7}{|l|}{$\mathrm{M} / \mathrm{T} F \mathrm{~F}(\mathrm{~Hz})$} \\
\hline 12 & $12.9 \pm 1.4$ & $9.3 \pm 1.4$ & $6.9 \pm 1.0$ & $16.4 \pm 2.0^{*}$ & $9.8 \pm 1.4$ & $8.3 \pm 1.1$ \\
\hline 16 & $17.9 \pm 1.7^{\dagger \dagger}$ & $12.0 \pm 1.2^{t \dagger}$ & $11.4 \pm 1.4^{t \dagger}$ & $24.1 \pm 2.1^{* * t \dagger}$ & $14.2 \pm 1.6^{\dagger \dagger}$ & $12.0 \pm 1.7^{t \dagger}$ \\
\hline 20 & $20.0 \pm 1.8$ & $13.4 \pm 1.6$ & $12.2 \pm 1.2$ & $31.3 \pm 2.7^{* * \dagger \dagger}$ & $22.8 \pm 2.9^{* *+\dagger}$ & $15.3 \pm 1.3^{* * * \dagger}$ \\
\hline 24 & $21.8 \pm 2.7^{\dagger \dagger}$ & $15.3 \pm 1.7^{\dagger \dagger}$ & $13.2 \pm 1.4^{t \dagger}$ & $34.4 \pm 2.8^{* * \dagger \dagger}$ & $27.6 \pm 2.9^{* * \dagger \dagger}$ & $21.3 \pm 2.6^{* *+\dagger}$ \\
\hline Range (Hz) & $11.2 \pm 1.8^{\dagger \dagger}$ & $6.3 \pm 0.6$ & $5.3 \pm 0.5$ & $21.0 \pm 2.2^{* *+\dagger}$ & $18.3 \pm 1.7^{* *}$ & $11.8 \pm 1.8^{* *}$ \\
\hline
\end{tabular}

Peak, phase-locked cortical firing rates ( $\mathrm{Hz}$ ) in response to simulated $\mathrm{M} / \mathrm{T}$ currents for the same conditions as in Table 2.

For each synapse type, the peak firing rates and range of firing rates were higher during $8 \mathrm{~Hz}$ rhythms than $2 \mathrm{~Hz}\left({ }^{*} p<0.05 * * * p<0.01\right.$, paired $t$ test). In addition, comparisons between cortical responses to discrete changes in presynaptic rates (i.e., 12 vs 16,16 vs 20,20 vs 24 ) yielded significant changes in both the 2 and $8 \mathrm{~Hz}$ cases $\left({ }^{\dagger \dagger} p<0.01\right.$, paired $t$ test). Finally, the range of mean cortical firing rates that represent the $12-24 \mathrm{~Hz}$ change in presynaptic rate was significantly greater in the Fynapse case $\left({ }^{+\dagger} p<0.01\right.$, unpaired $t$ test $)$ than the FD or D synapses.

neous firing rates of individual $\mathrm{M} / \mathrm{T}$ cells could be much higher $(10-100 \mathrm{~Hz})$ (Fig. 2 B). These average and instantaneous firing rates are consistent with odor-evoked changes in firing rate recorded in awake animals (Rinberg et al., 2006; Davison and Katz, 2007; Fuentes et al., 2008; Cury and Uchida, 2010; Shusterman et al., 2011).

To generate current stimuli to drive the cortical neurons, the individual $\mathrm{M} / \mathrm{T}$ spike trains were convolved with $\alpha$ function "synaptic" currents (Fig. 2C). For each M/T input, the synaptic currents were scaled based on the preceding IPI according to values of $\tau_{\text {fac }}$, and $\tau_{\text {rec }}$ that were comparable with the recorded values for F, FD, and D synapses (Table 1, Fig. 2C). Finally, these individual synaptic current waveforms were summed to create population excitatory currents ( $50 \mathrm{~s}$ duration) that were directly injected into cortical neurons (Fig. 2D,E). In the next section, we characterize the spike responses of cortical neurons to these simulated M/T population currents.

\section{Differential cortical responses with simulated $2 \mathrm{~Hz}$ versus 8 $\mathrm{Hz}$ respiratory rhythms}

To investigate how short-term plasticity and respiratory rhythms influence the cortical coding of presynaptic firing rates, we assessed the firing rates of cortical spike trains in response to our sinusoidally modulated $\mathrm{M} / \mathrm{T}$ population currents. The mean and phase-locked cortical firing rates, as well as statistical analyses for all combinations of synapse type, presynaptic rate, and simulated respiration frequency, are presented in Tables 2 and 3.

In Figure 3, we show examples of cortical spike trains in response to two different presynaptic firing rates $(16$ or $24 \mathrm{~Hz}$ ) that were modulated by $2 \mathrm{~Hz}$ (left) or $8 \mathrm{~Hz}$ (right) rhythms (Fig. 3A1,B1,C1). For each synapse type, F (reds), FD (greens), and $\mathrm{D}$ (blues), the cycle firing rate histograms show that the peak, phase-locked, cortical FRs increase significantly with presynaptic rate during $8 \mathrm{~Hz}$, but not $2 \mathrm{~Hz}$ rhythms (Fig. $\left.3 A 2, B 2, C 2 ;{ }^{* *} p<0.01\right)$. The cortical firing rates in response to all presynaptic rates are shown in Figure 4 . In the $8 \mathrm{~Hz}$ case, the peak, phase-locked FR increased significantly and linearly with presynaptic rate $(n=14 ; p<0.01$; Fig. $4 A 1-A 3)$. In contrast, during $2 \mathrm{~Hz}$ rhythms, peak cortical FR increased minimally for presynaptic rates $>16 \mathrm{~Hz}$ (Fig. 4A1-A3). This relationship was saturating and best fit by an exponential function $\left(\mathrm{X}^{2}, 0.12-0.29\right)$. Moreover, there was a broad range of cortical FRs $(\sim 12-20 \mathrm{~Hz})$ to represent changes in presynaptic rate in the $8 \mathrm{~Hz}$ case, but this range was significantly narrower in the $2 \mathrm{~Hz}$ case $(\sim 5-12 \mathrm{~Hz}$; Fig. 4 B2).
The mean firing rates of the cortical neurons increased linearly with presynaptic firing rate in both the 2 and $8 \mathrm{~Hz}$ cases (Fig. $4 C 1-C 3$ ). These increases were modest (range, $\sim 2-6 \mathrm{~Hz}$; Fig. $4 \mathrm{D} 2)$ compared with the range of presynaptic rates $(14 \mathrm{~Hz})$. In addition, the mean firing rates did not differ between the 2 and 8 $\mathrm{Hz}$ cases. Together, these results suggest that stimulus features represented by changes in presynaptic $\mathrm{M} / \mathrm{T}$ firing rates can be coded by changes in mean cortical firing rates regardless of respiration frequency $(2$ or $8 \mathrm{~Hz}$ ) as well as by spike timing relative to the respiratory cycle at active, sniff-like frequencies (i.e., $8 \mathrm{~Hz}$ ).

\section{Facilitation increases the gain of the input/output relationship in cortical neurons}

The relationship between cortical mean or phase-locked firing rates and presynaptic firing rate were qualitatively similar for all types of synapse. This was surprising given the extreme differences between facilitation-dominant and depression-dominant synaptic responses. However, there were quantitative differences in cortical firing rates in response to facilitating versus solely depressing inputs. F synapses promote higher phase-locked (Fig. $4 B$, Tables 2,$3 ;{ }^{* *} p<0.01$ ) and mean firing rates (Fig. $4 D$, Tables 2,$\left.3 ;{ }^{* *} p<0.01\right)$ than solely depressing inputs. Moreover, $\mathrm{F}$ and FD synapses give rise to a significantly greater range of peak and mean cortical firing rates that represent changes in presynaptic $\mathrm{M} / \mathrm{T}$ rate than depressing synapses $\left({ }^{* *} p<0.01\right.$; Fig. $4 B 2, D 2$, Tables 2, 3). Thus, the degree of facilitation expressed in M/T-tocortex synapses likely alters the gain of the relationship between input and output firing rates.

\section{Contributions of synaptic plasticity and respiratory rhythms to cortical responses}

To determine how plasticity and respiratory rhythm influence cortical responses, we took a step back look at how changes in presynaptic firing rate were reflected in the simulated M/T population currents. For each of the 24 sinusoidally modulated stimuli, we calculated the average current over a simulated respiratory cycle (Fig. 5A1-A3). For all synapse types (F, reds; FD, greens; D, blues), the peak current (in picoamperes) over a simulated respiratory cycle increased with increased presynaptic firing rate but did not differ for simulated breathing cycles $(2 \mathrm{~Hz})$ versus simulated sniffing cycles $(8 \mathrm{~Hz})$ (Fig. 5B1-B3). Facilitating inputs produced in higher peak currents and a greater range of current amplitudes to represent presynaptic rates (F synapses, 180-280 pA; FD synapses, 120-180 pA) compared with D synapses (110$160 \mathrm{pA})$. This increased drive likely underlies the higher mean 
A1
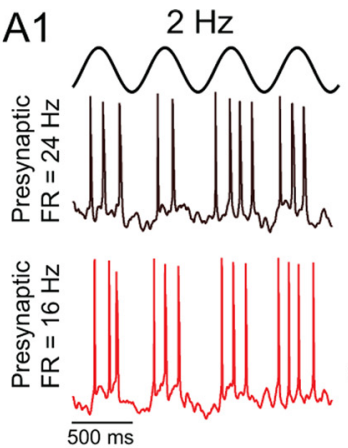

A2
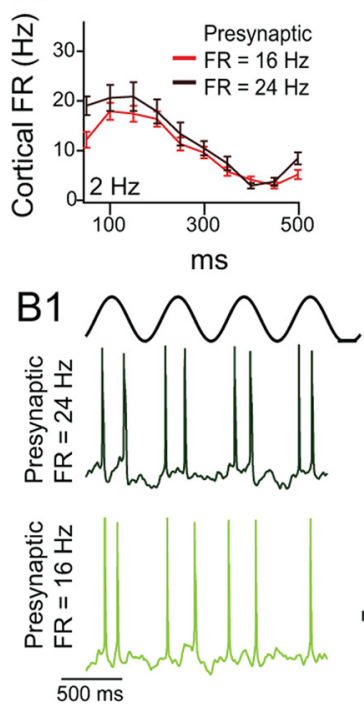

\section{B2}
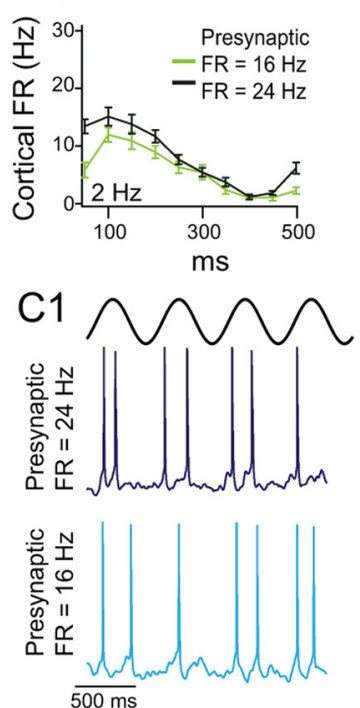

$\mathrm{C} 2$

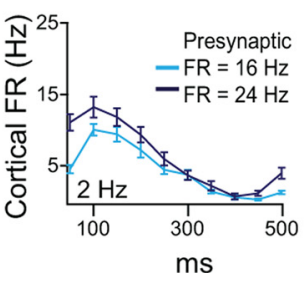

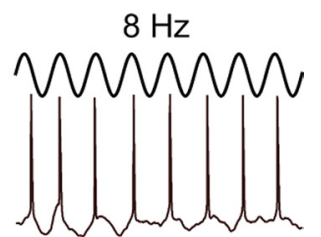
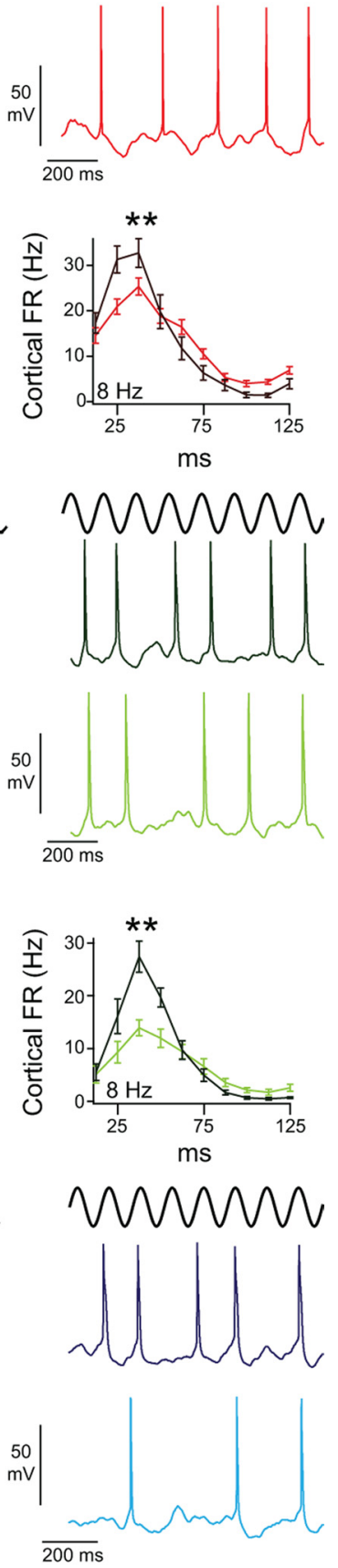

$\overline{200 \mathrm{~ms}}$

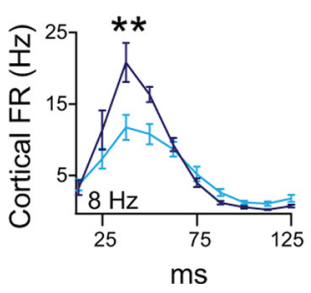

Figure 3. Cortical spike responses to simulated M/T population currents. $\boldsymbol{A 1}$, Representative pyramidal cell spike responses to simulated M/T population currents during $2 \mathrm{~Hz}$ (left) and $8 \mathrm{~Hz}$ and phase-locked cortical firing rates in response to facilitating inputs (F, FD) versus solely depressing inputs (Fig. 4, Tables 2, 3).

Changing the respiratory rhythm changes the rate at which odor inputs are delivered to the olfactory epithelium. Higher respiratory rates narrow the temporal window for $\mathrm{M} / \mathrm{T}$ population spiking relative to the cycle. In our simulated respiratory cycles, the window is narrowed from $500 \mathrm{~ms}(2 \mathrm{~Hz})$ to $125 \mathrm{~ms}(8$ $\mathrm{Hz}$ ), which has an organizing effect on spike times across the population and can increase the slope of the rising phase of the average synaptic current at the onset of the cycle. The slope of the rising phase was taken from 0 to $100 \mathrm{~ms}$ in the $2 \mathrm{~Hz}$ case and from 0 to $25 \mathrm{~ms}$ in the $8 \mathrm{~Hz}$ case. As expected, the slope of the current was greater $(1-3 \mathrm{pA} / \mathrm{ms})$ in the $8 \mathrm{~Hz}$ case than the $2 \mathrm{~Hz}$ case $(<1 \mathrm{pA} / \mathrm{ms})$. However, more importantly, the slope further increased linearly with presynaptic firing rate in the $8 \mathrm{~Hz}$ case but saturated in the $2 \mathrm{~Hz}$ case (Fig. 5C1-C3). These results are reminiscent of the relationship between phase-locked cortical FRs and presynaptic firing rate in the $8 \mathrm{~Hz}$ case versus $2 \mathrm{~Hz}$ case (Fig. 4).

Although changes in respiratory rhythm produce population current slopes that are generally greater in the $8 \mathrm{~Hz}$ case than the $2 \mathrm{~Hz}$ case, they do not fully explain the differential sensitivity of slope or cortical FR to changes in presynaptic rate. We next explored how synaptic plasticity might contribute to the relative insensitivity to changes in presynaptic rate in the $2 \mathrm{~Hz}$ case. We generated population currents of neutral $(\mathrm{N})$ (neither facilitating nor depressing) synaptic inputs. The population of M/T spike trains were simulated as previously described for F, FD, or D synapses except these were convolved with $\alpha$ function synaptic currents with amplitudes (20 pA) that did not vary with interpulse interval. As seen in the F, FD, and D cases, the maximum current attained over the cycle increased with increasing presynaptic firing rate and did not differ for $2 \mathrm{~Hz}$ versus $8 \mathrm{~Hz}$ cycles (Fig. $6 A, B)$. These current amplitudes (135-225 pA) were lower than those of $\mathrm{F}$ synapses but greater than FD or D synapses (compare with Fig. $5 B$ ). In the $8 \mathrm{~Hz}$ case, the slope of the rising phase of the neutral currents increased linearly from 0.5 to $2.5 \mathrm{pA} / \mathrm{ms}$ with presynaptic rate similar to F, FD, and D currents (Fig. 6C). However, in the $2 \mathrm{~Hz}$ case, the slope of the neutral currents also linearly increased from 0.1 to $1 \mathrm{pA} / \mathrm{ms}$ with presynaptic rate (Fig. $6 D$, black circles), which contrasts with saturating slope values for F, FD, and D synapses (Fig. 6D). Thus, synaptic plasticity contributes substantially to the relationship between current slope and presynaptic firing rate.

\section{Relationship between respiratory frequency, presynaptic firing rates, and synaptic scale}

In the previous sections, we show that cortical neurons respond differentially to changes in presynaptic firing rate when simulated M/T currents are modulated at $2 \mathrm{~Hz}$ (breathing) versus $8 \mathrm{~Hz}$ (sniffing) frequencies. We also show that both simulated respiration frequency and short-term plasticity contribute to cortical responses. However, it remains to be determined how synaptic

(right) sinusoidal rhythms for two different presynaptic FRs: $16 \mathrm{~Hz}$ (bottom trace) and $24 \mathrm{~Hz}$ (top trace). Responses are shown for simulated population inputs scaled by facilitation-dominant $(\mathrm{F})$ synapses (reds). $\boldsymbol{A 2}$, Average firing rate cycle histograms for cortical neurons $(n=14)$ during 2 $\mathrm{Hz}$ (left) or $8 \mathrm{~Hz}$ (right) modulation. The peak, phase-locked, cortical FR in the $8 \mathrm{~Hz}$ case was significantly greater in response to $24 \mathrm{~Hz}$ versus $16 \mathrm{~Hz}$ presynaptic firing rates $\left(^{* *} \mathrm{p}<0.01\right.$, paired $t$ test). The peak cortical firing rate did not differ during $2 \mathrm{~Hz}$ rhythms for presynaptic firing rates between 16 and $24 \mathrm{~Hz}$. B1, B2, As in $\mathbf{A} 1$ and $\boldsymbol{A} \mathbf{2}$ but for facilitating-depressing (FD) synapses (greens). C1, C2, As in $\boldsymbol{A} \mathbf{1}$ and $\boldsymbol{A} \mathbf{2}$ but for depression-dominant (D) synapses (blues). Error bars indicate SEM. 
A1
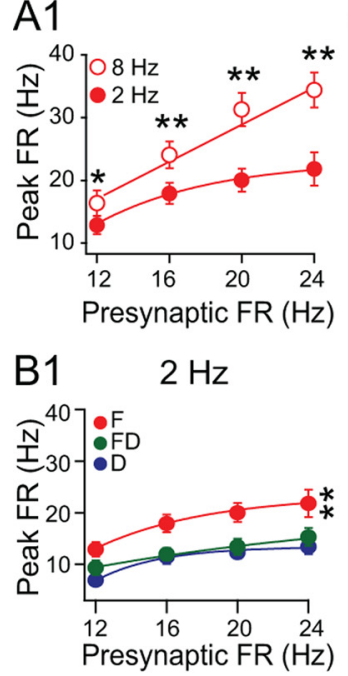

C1
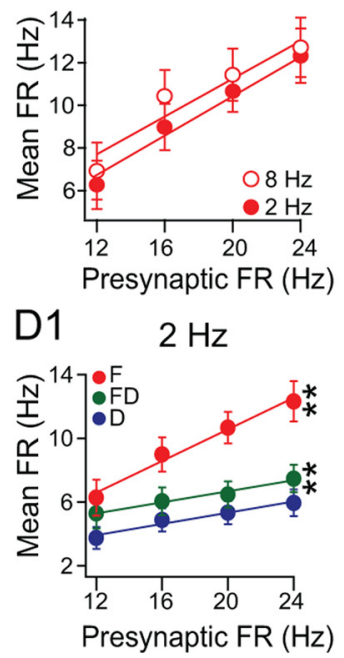

A2

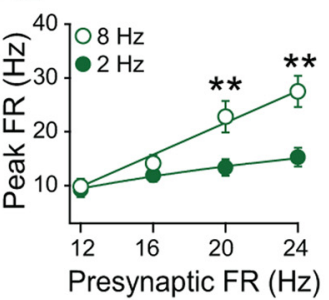

$8 \mathrm{~Hz}$

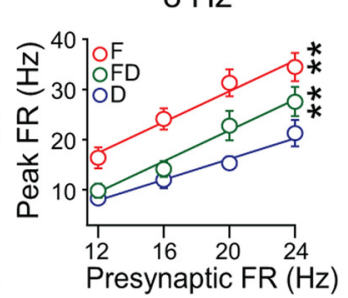

C2

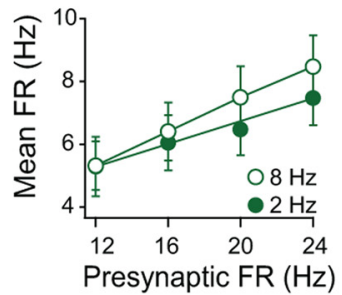

$8 \mathrm{~Hz}$

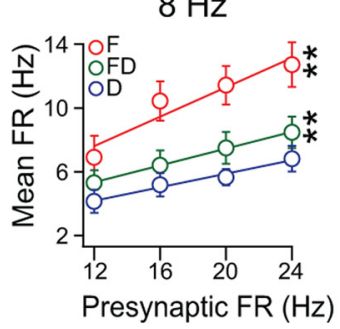

A3

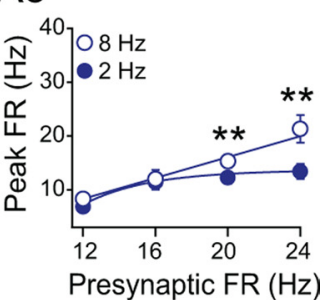

B2

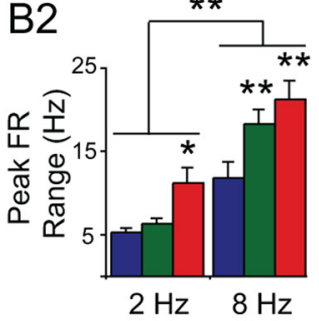

C3

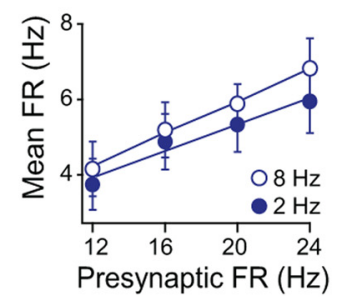

D2

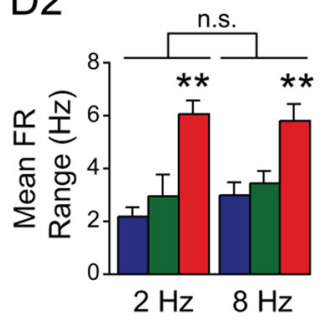

Figure 4. Summary of cortical spike responses to simulated M/T population currents. $A$, The peak, phase-locked, cortical FRs for all presynaptic firing rates. Peak FR significantly differed between $2 \mathrm{~Hz}$ (solid circles) and $8 \mathrm{~Hz}$ modulations (open circles; ${ }^{* *} p<$ 0.01 , paired $t$ test). The relationship between peak FR and presynaptic FR was linear in the $8 \mathrm{~Hz}$ case $(R>0.85)$ but saturating in the $2 \mathrm{~Hz}$ case (exponential fit, $\mathrm{X}^{2}: 0.12-0.29$ ). This was true for all synapse types: $F$ synapses, red $(\boldsymbol{A 1}) ; \mathrm{FD}$ synapses, green $(\boldsymbol{A 2}) ; \mathrm{D}$ synapses, blue (A3). $\boldsymbol{B} \mathbf{1}$, In both the $2 \mathrm{~Hz}$ (left) and $8 \mathrm{~Hz}$ (right) cases, F synapses drove cortical responses with higher peak FRs than $\mathrm{FD}$ or $\mathrm{D}$ synapses ${ }^{* *} p<0.01$, paired $t$ test). $\mathbf{B 2}$, The range of peak cortical $F R$ was greatest for $\mathrm{F}$ synapses during $2 \mathrm{~Hz}$ rhythms and for $\mathrm{F}$ and $\mathrm{FD}$ synapses during $8 \mathrm{~Hz}$ rhythms (F, red bars; $\mathrm{FD}$, green bars; $\mathrm{D}$, blue bars; ${ }^{*} p<0.05$, ${ }^{* *} p<0.01$, paired $t$ test; range, cortical FR for the highest presynaptic FR minus the cortical FR for the lowest presynaptic FR). C, The mean FRs increased with presynaptic rate but did not differ for $2 \mathrm{~Hz}$ (solid circles) versus $8 \mathrm{~Hz}$ (open circles) modulations, F synapses (C1), FD synapses (C2), D synapses (C3). D1, In both the $2 \mathrm{~Hz}$ (left) and $8 \mathrm{~Hz}$ (right) cases, F and FD synapses drove cortical responses with higher mean FRs than D synapses $(* * p<0.01$, paired $t$ test). D2, The range of mean cortical FRs was greatest for facilitation-dominant synapses $(F$, red bars; ${ }^{* *} p<0.01$, paired $t$ test) (Tables 2,3 ). For all synapse types, the range of mean firing rates did not significantly (n.s.) differ between the 2 and $8 \mathrm{~Hz}$ cases. Error bars indicate \pm SE.

plasticity contributes to saturating current slopes and, consequently, invariant phase-locked cortical firing rates during $2 \mathrm{~Hz}$ but not $8 \mathrm{~Hz}$ rhythms. This phenomenon occurs for all synapse types, so it is unlikely that facilitation, which is only expressed in $\mathrm{F}$ and FD synapses, is the primary cause. For this reason, we focus on a role for short-term depression in mediating saturating cortical responses during $2 \mathrm{~Hz}$ modulations.

In general, as presynaptic firing rate increases, more synaptic efficacy is used $(u)$ and there is less recovery of the remainder $(r)$ between pulses (Fig. $1 D$, yellow highlight). This enhanced depression decreases overall synaptic scale and could counter the increases in synaptic drive produced by higher presynaptic M/T

firing rates. Such a mechanism requires substantial overlap between the timing for increased presynaptic spike activity and that of decreased synaptic scale (recruitment of depression). To ascertain the temporal relationship between presynaptic spike activity and changes in synaptic scale, we plot the normalized M/T spike rate and synaptic scale against the phase $(\phi)$ of the simulated respiratory cycle between 0 and $2 \pi$ (Fig. 7A1,A2). When presynaptic inputs were modulated by $2 \mathrm{~Hz}$ rhythms, the time course of synaptic scale (F synapses, red; FD synapses, green; D synapses, blue) is inversely related to $\mathrm{M} / \mathrm{T}$ spike rate (black) — as spike rate increases, synaptic scale decreases (Fig. 7A1). The phase difference $(\Delta \phi)$ between the peak of the M/T spike rate and the trough of synaptic scale (maximum depression) is small, $0.2 \pi$ (Fig. 7A1). Thus, when M/T spike rate is maximal, synaptic amplitude is nearly minimal (scale, $\sim 0.10$ ). This suggests that, during the $2 \mathrm{~Hz}$ cycle, recruited depression is optimally timed to cancel increases in $\mathrm{M} / \mathrm{T}$ rates resulting in saturating cortical responses. In contrast, when population currents were modulated by 8 $\mathrm{Hz}$ rhythms, synaptic scale peaks early in the cycle and M/T spikes have a high probability of arriving at a time when depression is weak and synaptic amplitudes are high (scale, 0.5-1; Fig. 7A2). Furthermore, the phase difference between the peak spike rate and the trough of the synaptic scale is greater $(\Delta \phi=0.6 \pi)$ than in the $2 \mathrm{~Hz}$ case. Since the majority of M/T inputs arrive before synaptic scale is minimized, these inputs escape substantial depression and ultimately drive cortical spike responses that can code increases in presynaptic $\mathrm{M} / \mathrm{T}$ firing rates.

In rodents, respiratory frequencies vary from 1 to $12 \mathrm{~Hz}$, so we explored the temporal relationship between $\mathrm{M} / \mathrm{T}$ spikes and synaptic scale over a range of simulated respiratory rhythms. At modulation frequencies consistent with passive respiration $(1-4 \mathrm{~Hz})$, we found that the phase difference between the trough of the synaptic scale and the peak presynaptic firing rate is small $(\Delta \phi=0.2 \pi)$. This suggests a substantial temporal overlap between presynaptic spiking and the recruitment of depression that can counter increases in firing rate. However, the phase difference substantially increases $(\Delta \phi=0.4-$ $0.6 \pi)$ in a nearly step-like fashion with the transition to snifflike, modulation frequencies ( $\geq 5 \mathrm{~Hz}$; Fig. $7 B$, yellow box). This phase difference increases the probability that presynaptic spikes will drive cortical responses before depression is recruited. These results suggest that the transition from passive respiration to active sniffing creates a window of opportunity for $\mathrm{M} / \mathrm{T}$ inputs to drive cortical responses that code stimulus information in phase-locked firing rates. 

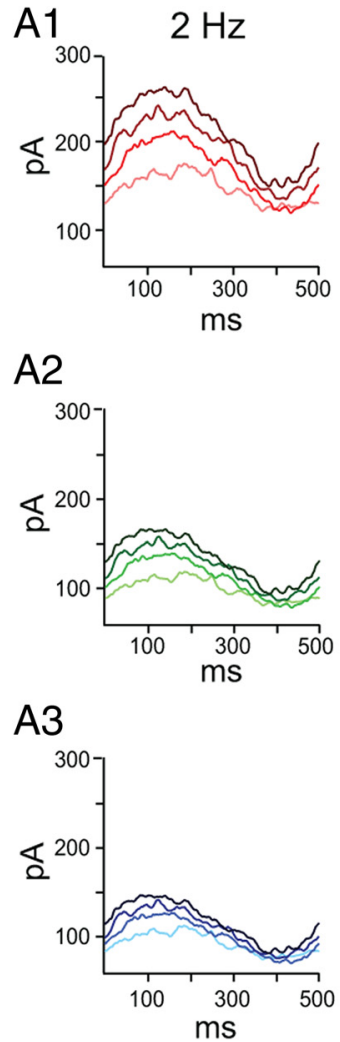

$8 \mathrm{~Hz}$
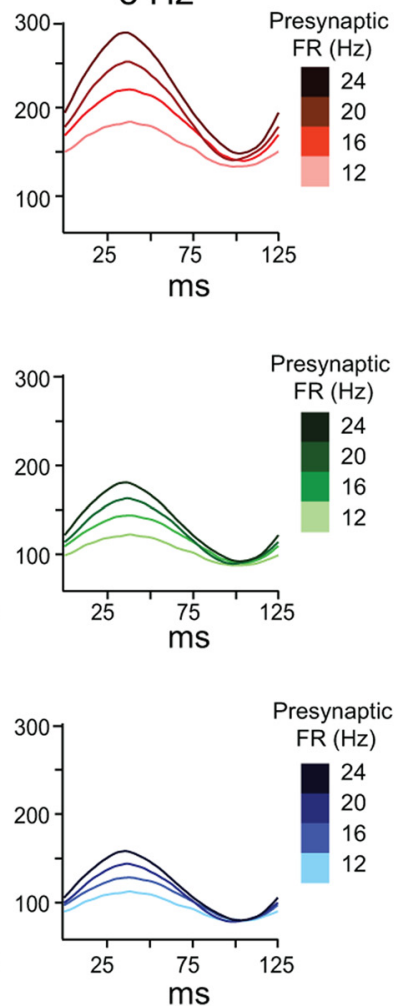

B1

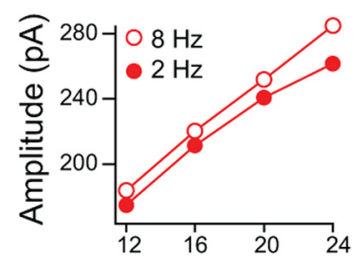

Presynaptic FR $(\mathrm{Hz})$

B2

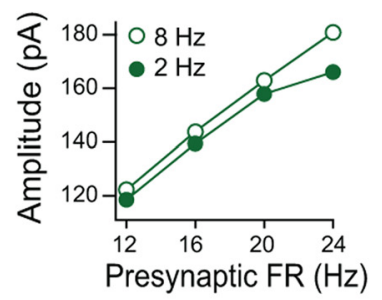

B3

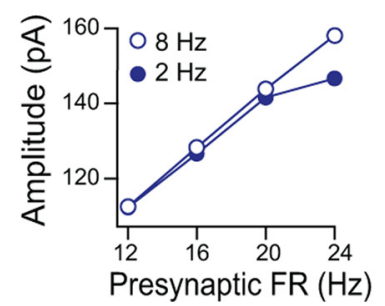

C1

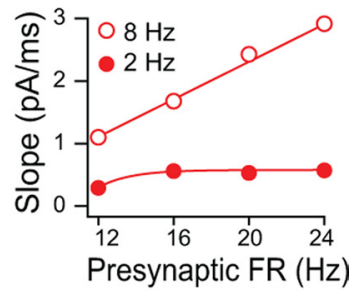

C2

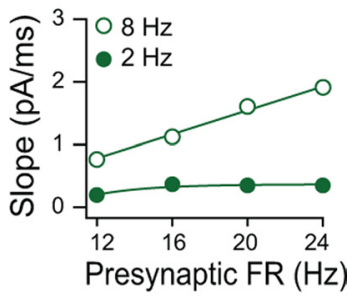

C3

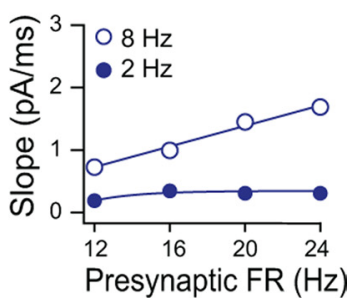

Figure 5. Analysis of the amplitude and slope of simulated population currents. $A$, Average current (in picoamperes) over the cycle for simulated $\mathrm{M} / \mathrm{T}$ population inputs modulated at $2 \mathrm{~Hz}$ (left) and $8 \mathrm{~Hz}$ (right) is shown for each presynaptic FR (12-24 Hz) and each synapse type: facilitation dominant (F) (reds) (A1); facilitating-depressing (FD) (greens) (A2); depression dominant (D) (blues) (A3). B, Maximum current amplitude during $2 \mathrm{~Hz}$ (solid circles) and $8 \mathrm{~Hz}$ (open circles) cycles for each presynaptic firing rate for $\mathrm{F}$ synapses, red (B1); $F$ synapses, green (B2); and D synapses, blue (B3). C, The slope of the rising phase of the current plotted against presynaptic rate. The slope was measured for the first $0-100 \mathrm{~ms}(2 \mathrm{~Hz})$ or $0-25 \mathrm{~ms}(8 \mathrm{~Hz})$ of the currents shown in $A$. In the $8 \mathrm{~Hz}$ case, the relationship between slope and presynaptic rate was linearly fit in the $8 \mathrm{~Hz}$ case (open circles) but exponentially fit in the $2 \mathrm{~Hz}$ case (closed circles). C1, F synapses, red. C2, FD synapses, green. C3, D synapses, blue.
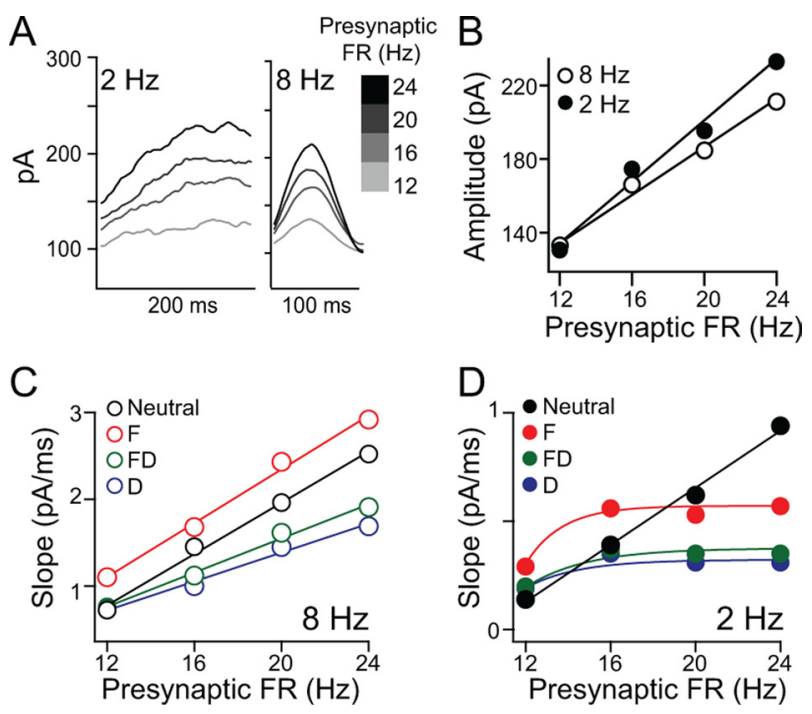

Figure 6. Contribution of synaptic plasticity to simulated population currents. $A$, Average current (in picoamperes) across cycles for simulated $M / T$ population inputs with neutral (N) synapses modulated at $2 \mathrm{~Hz}$ (left) and $8 \mathrm{~Hz}$ (right). $\boldsymbol{B}$, Maximum current amplitude attained during $2 \mathrm{~Hz}$ (solid circles) and $8 \mathrm{~Hz}$ (open circles) cycles plotted against the presynaptic firing rate for $\mathrm{N}$ synapses. $\boldsymbol{C}, \boldsymbol{D}$, The slope of the rising phase of the population current for $\mathrm{N}$ synapses (black), F synapses (red), FD synapses (green), and D synapses (blue) during $8 \mathrm{~Hz}$ (C, open circles) or $2 \mathrm{~Hz}$ (D, closed circles) rhythms.
Cortical responses to simulated $\mathrm{M} / \mathrm{T}$ burst firing delivered at $2 \mathrm{~Hz}$ versus $8 \mathrm{~Hz}$ rhythms

In the previous sections, sinusoidal modulations of presynaptic firing rate provide an intuitive explanation for how the interactions between the timescales of respiratory rhythms and shortterm synaptic plasticity might influence cortical coding. We questioned whether these observations would be maintained in response to more realistic, burst-like, $\mathrm{M} / \mathrm{T}$ firing patterns recorded in vivo. Based on cycle histograms of $\mathrm{M} / \mathrm{T}$ firing rates recorded during passive respiration (Carey and Wachowiak, 2011) and active sniffing (Cury and Uchida, 2010) in vivo, we created two galleries of firing patterns that simulated the "bursty" spike trains of M/T cells when odors are sampled at $2 \mathrm{~Hz}$ (Fig. $8 \mathrm{Al}$ ) or $8 \mathrm{~Hz}$ (Fig. $8 \mathrm{B1}$ ). For each population current stimulus, we randomly chose 20 patterns from a given gallery and jittered the onset of each pattern by $10 \pm 5 \mathrm{~ms}$. This ensured that the population of simulated $\mathrm{M} / \mathrm{T}$ spikes tiled the respiratory cycle (Fig. 8A2,B2) as previously described (Cury and Uchida, 2010; Shusterman et al., 2011). These patterns were scaled by three different peak firing rates with means of 150,200 , or $250 \mathrm{~Hz}$ $(\mathrm{SD} \pm 50 \mathrm{~Hz})$. We then used these patterns to drive Poisson distributed spike times (see Materials and Methods). The resulting spike trains were convolved with $\alpha$ function currents that were scaled by synaptic plasticity as previously described. Finally, the summed population currents were injected at the somas of cortical pyramidal cells $(n=5)$.

The characteristics of these stimuli and the elicited cortical responses were very similar to those described previously for si- 

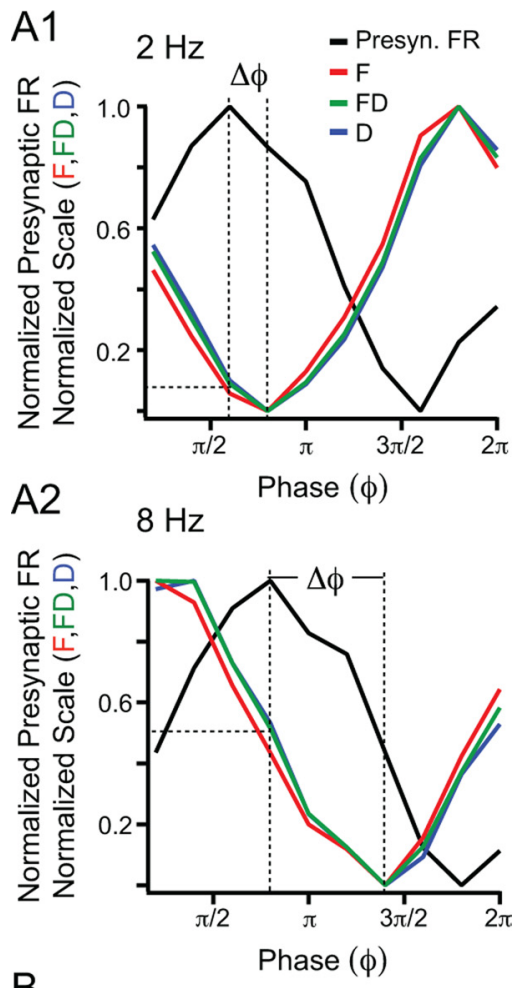

B

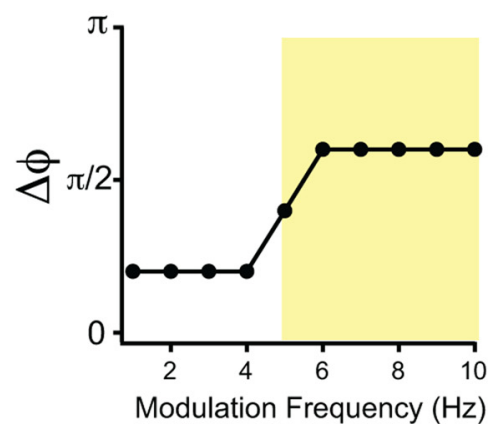

Figure 7. Relationship between simulated respiration frequency, M/T spike times, and synaptic plasticity. $\boldsymbol{A}$, Phase relationship between presynaptic FRs and synaptic scale over the simulated respiratory cycle. Cycle time was normalized to phase $(\phi)$ between 0 and $2 \pi$, and $\Delta \phi$ was the phase difference between the trough of the synaptic scale and the peak, presynaptic firing rate. The presynaptic firing rates (black) and synaptic scale for all synapse types ( $F$, red; $F D$, green; D, blue) were normalized between 0 and $1 . \boldsymbol{A 1}$, In the $2 \mathrm{~Hz}$ case, synaptic scale decreases as presynaptic rate increases, and there is a small phase difference $(\Delta \phi=0.2 \pi$; vertical dashed lines) between the peak firing rate and trough of the synaptic scale. When firing rate is maximal (1), scale is nearly minimal ( $\sim 0.10$; horizontal dashed line). $\mathbf{A 2}$, In the $8 \mathrm{~Hz}$ case, both synaptic scale and presynaptic rate peak early in the cycle with phase difference, $\Delta \phi=$ $0.6 \pi$ (vertical dashed lines). When firing rates peak, synaptic scale is $\sim 0.5$ (horizontal dashed line). $\boldsymbol{B}$, Phase difference $(\Delta \phi)$ between synaptic scale and presynaptic firing rate for simulated respiration modulation frequencies between 1 and $10 \mathrm{~Hz}$. At simulated sniff frequencies $(>5$ $\mathrm{Hz}$; yellow box), there is a step-like increase in $\Delta \phi$.

nusoidally modulated firing rates. The peak cortical FR of the cycle histograms did not vary with presynaptic rate during $2 \mathrm{~Hz}$ rhythms but increased during $8 \mathrm{~Hz}$ rhythms (Fig. 8A4,B4,C1$C 3)$. Indeed, for all synapse types, the differences in peak cortical FR observed with sinusoidally modulated inputs delivered at $2 \mathrm{~Hz}$ versus $8 \mathrm{~Hz}$, appear amplified by the use of realistic M/T firing patterns (Fig. 8C1-C3). Moreover, facilitation greatly enhanced the gain of these input-output relationships. As we have shown previously, the slope of the average current across cycles saturated with increasing presynaptic rate for all synapse types during $2 \mathrm{~Hz}$ cycles (Fig. $8 A 3, D 1)$ but increased with presynaptic rate during 8 $\mathrm{Hz}$ cycles (Fig. 8 B3,D2). Furthermore, in the $2 \mathrm{~Hz}$ case, synaptic scale, which is minimized when presynaptic spike rates are maximized ( $\Delta \phi=0 \pi$; Fig. $8 E 1$, dashed line), can directly counteract changes in presynaptic activity. In the $8 \mathrm{~Hz}$ case, the recruitment of depression is delayed ( $\Delta \phi=0.4 \pi$; Fig. $8 E 2$, dashed lines) with respect to presynaptic spiking, creating a window of opportunity to drive cortical responses. Together, these results suggest that short-term synaptic plasticity can modulate cortical responses recorded in vivo during passive respiration or active sniffing.

\section{Discussion}

In this study, we investigated the interaction between behavioral and synaptic timescales that influence cortical activation and stimulus coding in the mouse olfactory system. The transition from passive breathing $(1-4 \mathrm{~Hz})$ to active sniffing $(5-12 \mathrm{~Hz})$ is a critical olfactory behavior, yet the influence of sniffing on the activation of cortical neurons during odor coding has not been fully elucidated. We have identified multiple timescales for shortterm facilitation and depression at synapses between mitral/ tufted cells and excitatory cortical neurons. We have shown that interactions between the timing of short-term synaptic depression and simulated respiratory rhythms produce significant differences in the cortical firing rates during $2 \mathrm{~Hz}$ (breathing) versus $8 \mathrm{~Hz}$ (sniffing) modulations. Specifically, during $8 \mathrm{~Hz}$ modulations, increases in presynaptic activity are coded by increases in the phase-locked firing rates of cortical neurons. This contrasts with saturating, phase-locked firing rates during $2 \mathrm{~Hz}$ modulations. We also show that the gain of these responses is modulated by short-term facilitation. Together, our results suggest that the differential recruitment of short-term plasticity by transitioning from passive breathing to active sniffing shapes the transfer and coding of odor information between the olfactory bulb and cortex.

\section{Respiratory rhythms, short-term depression, and cortical coding}

Previous studies have suggested that short-term facilitation and depression may contribute to the differential response properties of semilunar versus pyramidal cells (Suzuki and Bekkers, 2006, 2011). Here, we suggest a new function in which short-term synaptic depression interacts with timescales of respiratory rhythms to alter cortical coding. For slow respiratory rhythms $(<4 \mathrm{~Hz})$, the temporal overlap between presynaptic spike times and the recruitment of depression produces cortical spike responses that saturate with changes in presynaptic firing rate. During highfrequency, sniff-like rhythms $(>5 \mathrm{~Hz})$, presynaptic spikes occur early in the cycle when synaptic depression is relatively weak. This creates a window of opportunity when changes in presynaptic rate can be coded by phase-locked cortical firing rates before depression is maximally recruited. Moreover, since all synapse types (F, FD, and D) express depression, the mechanism by which cortical responses are modulated by increases in respiratory rhythm may be common to pyramidal and semilunar cells.

In our sinusoidally modulated model, presynaptic spike timing relative to the respiratory cycle changes with increases in firing rate and simulated respiration frequency. These changes in spike timing contribute to the slope of the population currents that drive phase-locked spikes in cortical neurons during snifflike rhythms. Although $\mathrm{M} / \mathrm{T}$ spike timing relative to the respiratory cycle changes with odor concentration and firing rate in vivo (Cang and Isaacson, 2003; Carey and Wachowiak, 2011), the impact of respiratory frequency on spike timing remains unre- 
A1
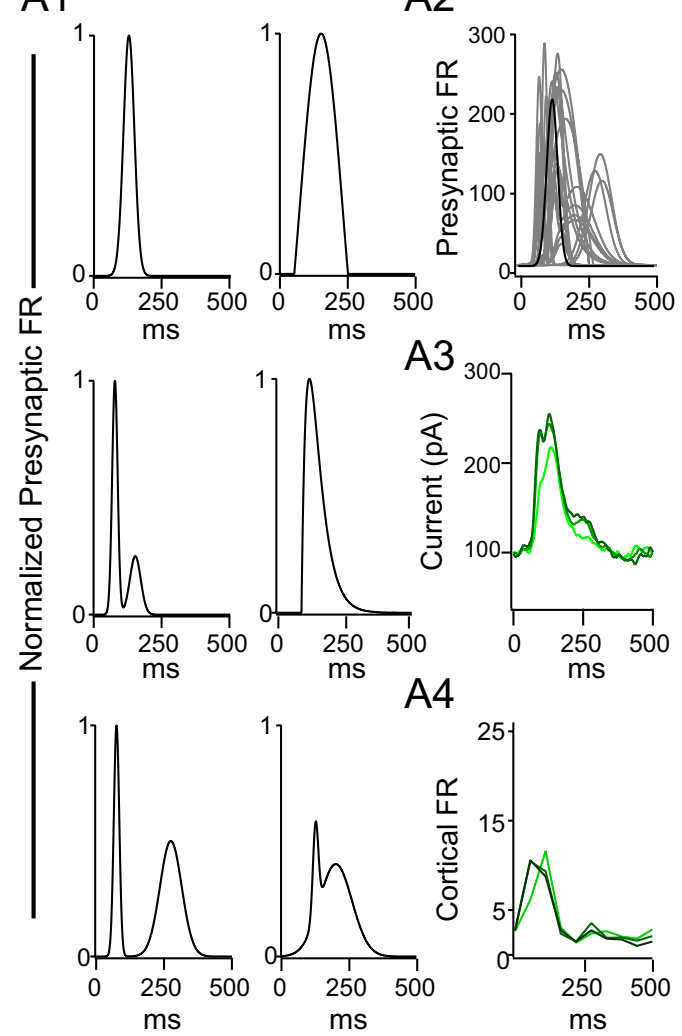

D1

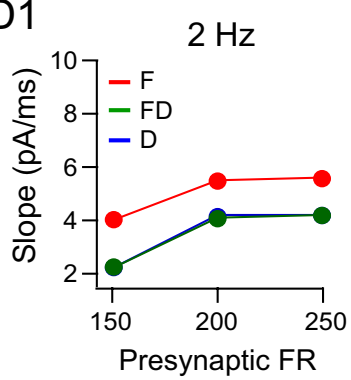

A4

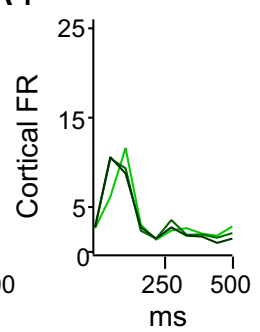

A2

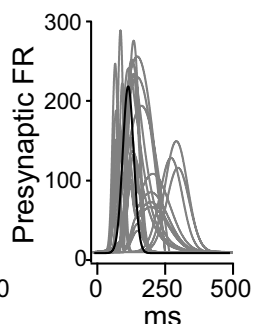

D2

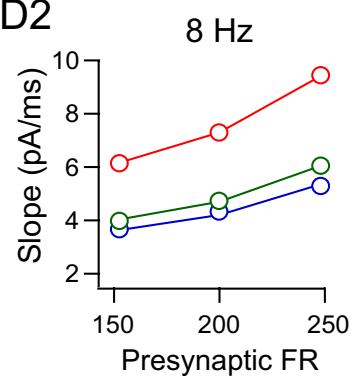

B1
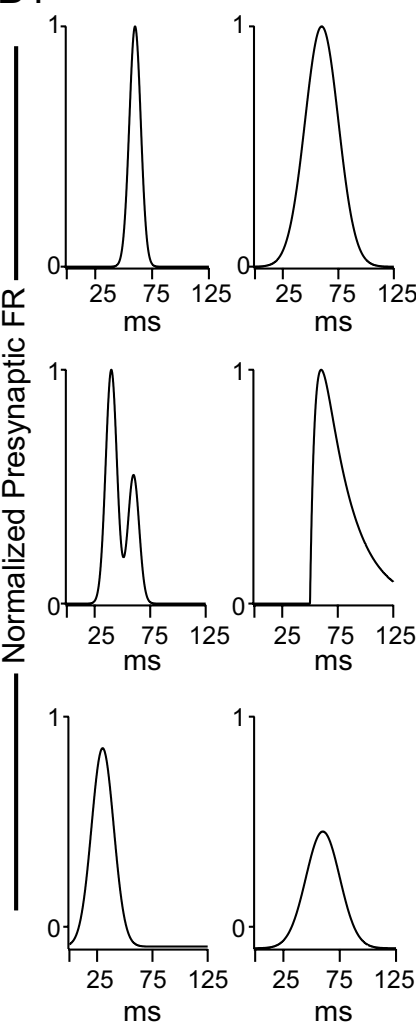

B2

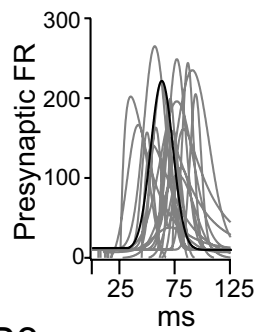

B3

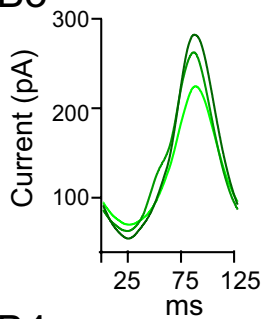

B4

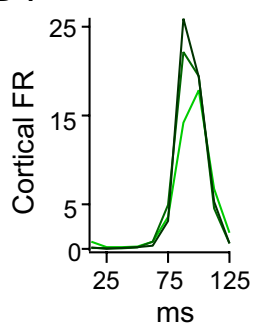

C1
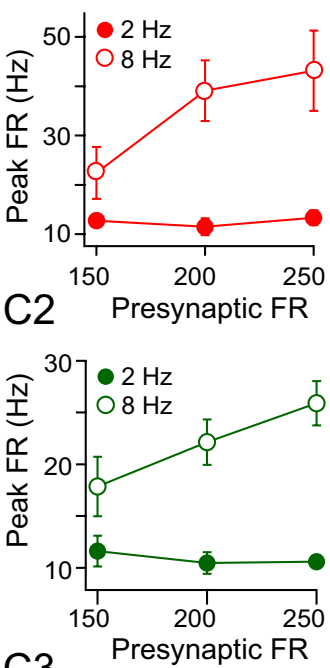

C3

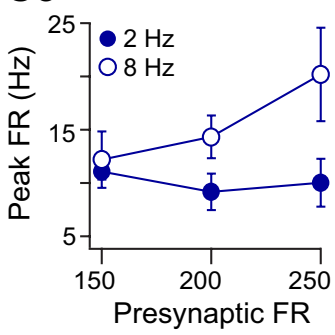

E1

$2 \mathrm{~Hz}$
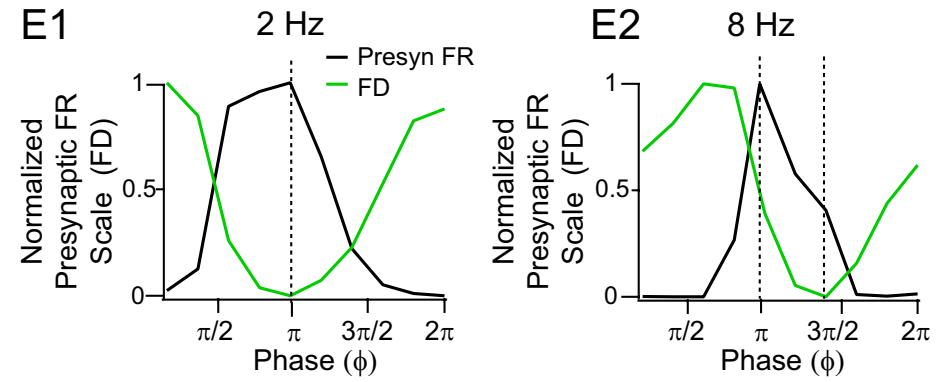

Figure 8. Cortical responses to simulated, burst-like M/T inputs. A1, Samples from a gallery of burst-like PSTHs based on M/T cells recorded in vivo during $2 \mathrm{~Hz}$ respiratory rhythms (Carey and Wachowiak, 2011). A2, Twenty cycle histograms that were randomly drawn from the gallery and scaled to a peak FR of $200 \pm 50 \mathrm{~Hz}$. The onsets were jittered $10 \pm 5 \mathrm{~ms}$. $\mathbf{A 3}$, Cycle triggered average of the population current for FD synapses driven at presynaptic rates of $150 \mathrm{~Hz}$ (light green), $200 \mathrm{~Hz}$ (green), and $250 \mathrm{~Hz}$ (dark green). A4, Cycle histograms of cortical firing rates in response to the population stimuli for the different presynaptic firing rates, $150 \mathrm{~Hz}$ (light green), $200 \mathrm{~Hz}$ (green), and $250 \mathrm{~Hz}$ (dark green). B1-B4, Same as in A1-A4 except the modulation frequency was $8 \mathrm{~Hz}$, and the PSTH gallery was based on in vivo recordings during sniffing (Cury and Uchida, 2010).C, The peak, phase-locked, cortical firing rates for all presynaptic firing rates were greater and increased with presynaptic rate in response to $8 \mathrm{~Hz}$ rhythms (open circles) versus saturating responses during $2 \mathrm{~Hz}$ rhythms (solid circles): F synapses, red (C1); FD synapses, green (C2); D synapses, blue (C3). Error bars indicate SEM. D. The slope of the rising phase of the cycle triggered average of the population current for each presynaptic rate. For all synapse types - F synapses (red), FD synapses (green), and D synapses (blue) - the relationship between slope and presynaptic rate saturated in the $2 \mathrm{~Hz}$ case (D1, closed circles) but increased in the $8 \mathrm{~Hz}$ case (D2, open circles). $\boldsymbol{E}$, Phase relationship between presynaptic FRs (black) and synaptic scale (shown for FD synapse; green) over the simulated respiratory cycle. Cycle time was normalized to phase $(\phi)$ between 0 and $2 \pi$. E1, Two hertz rhythm. E2, Eight hertz rhythm.

solved. It has been shown that the phase of M/T spiking does scale with respiration frequency during repetitive sniffing (Carey and Wachowiak, 2011; Shusterman et al., 2011). However, it has also been shown that, on the first respiratory cycle of an odor response, absolute spike timing relative to the onset of inspiration does not differ for breathing versus sniffing frequencies (Cury and Uchida, 2010; Carey and Wachowiak, 2011). Nonetheless, when our model incorporated realistic M/T firing patterns based on recordings during breathing (Carey and Wachowiak, 2011) and sniffing (Cury and Uchida, 2010), differential cortical coding with respiratory frequency is maintained. This suggests that the timescales of synaptic plasticity and respiration can play an important role in how cortical neurons code increases in bulbar activity in vivo. Future studies in awake animals that investigate the coding of changes in odor features by $\mathrm{M} / \mathrm{T}$ and cortical neurons at different respiration frequencies will be essential to verify these predictions.

\section{Short-term facilitation at M/T-to-cortical neuron synapses}

We have classified three types of synaptic input between M/T neurons and cortical principal cells that differ in their expression of short-term facilitation and depression. All synaptic inputs expressed frequency-dependent depression; however, only two types expressed facilitation. Consistent with previous studies, facilitating inputs were biased toward bursting, pyramidal neurons, while regular-spiking semilunar neurons received solely depress- 
ing input (Bower and Haberly, 1986; Hasselmo and Bower, 1990; Suzuki and Bekkers, 2006, 2011). However, we found that facilitating inputs could be further classified as strongly facilitating, $\mathrm{F}$ synapses, or moderately facilitating, FD synapses. The time constants for facilitation and depression of these two types of synapses differed significantly and produced dramatically different cortical responses.

Our results suggest that the functional impact of facilitation in LOT inputs may distribute along a continuum that depends on the balance facilitation and depression. Although synapses that expressed facilitation (F, FD) were initially weaker in amplitude than solely depressing synapses, population currents generated by $\mathrm{F}$ synapses resulted in the highest cortical firing rates and the greatest range of rates to represent presynaptic activity. Currents composed of FD synapses evoked midrange cortical responses, while D synapses produced the lowest firing rates and the narrowest range of responses. Thus, the degree of facilitation in LOT synapses may play an important role in enhancing the gain of the cortical input/output relationship and/or broadening the range of cortical responses (Abbott et al., 1997).

The range of synaptic responses we observe highlights the fact that the mechanism of target-dependent, short-term plasticity at the $\mathrm{M} / \mathrm{T}$ to cortex synapses is currently unknown. One possibility is that single $\mathrm{M} / \mathrm{T}$ axons make different types of synaptic connections depending on the identity of the postsynaptic cell as in other cortical areas (Markram et al., 1998; Reyes et al., 1998). Alternatively, mitral or tufted cells may preferentially target one type of principal neuron or cortical area (Nagayama et al., 2010). A third possibility is that cortical neurons receive a mix of inputs that vary in amplitude (Franks and Isaacson, 2006) and balance of facilitation or depression. Although our use of minimal stimulation aims to activate just one or a few axons, this third possibility cannot be entirely ruled out. Future studies that focus on the anatomical and functional specificity of connections between the olfactory bulb and the cortex are essential to resolve this issue.

\section{Potential interactions between LOT inputs and cortical circuitry}

Cortical activation by dynamic LOT synapses is likely further modulated by feedforward inhibition (Luna and Schoppa, 2008; Poo and Isaacson, 2009; Stokes and Isaacson, 2010). Previous studies of synaptic plasticity at afferent synapses have blocked inhibition to isolate excitatory responses (Franks and Isaacson, 2006; Suzuki and Bekkers, 2006, 2011). We chose to keep inhibition intact and therefore cannot rule out the possibility that recruitment of feedforward inhibition contributes to the plasticity dynamics we observe. We routinely depolarized the cortical neurons to ensure that the PSPs did not reverse and were thus predominantly excitatory. In some neurons, LOT stimulation yielded EPSPs followed or obscured by strong inhibitory PSPs (Luna and Schoppa, 2008). These neurons were not included in our analyses. Nonetheless, given that LOT synapses onto L1 interneurons also depress (Stokes and Isaacson, 2010), the mechanisms described for differential phase-locked responses with respiratory frequency in excitatory neurons may also apply to spike activity in these inhibitory neurons. Moreover, the time lag between the recruitment of excitation and inhibition (Luna and Schoppa, 2008; Poo and Isaacson, 2009; Stokes and Isaacson, 2010) may further enhance cortical phase locking during highfrequency respiration. Finally, the recruitment of inhibition may counter or enhance the changes in gain mediated by the different types of facilitation at excitatory synapses (Mitchell and Silver, 2003; Arevian et al., 2008; Ferrante et al., 2009). Our study serves as a starting point for predictions about how synaptic plasticity at LOT synapses influences cortical responses. As more information becomes available, our model could be amended to include interactions between respiratory frequency and the timescales of feedforward inhibition and/or recurrent cortical circuits.

\section{Sniffing behavior and cortical coding}

Once an odor is detected, animals can often make learned discriminations or alter behavior in one or two sniffs $(\sim 150-200$ ms) (Uchida and Mainen, 2003; Kepecs et al., 2007; Wesson et al., 2008a), suggesting a short temporal window for cortical processing before changes in behavior (Wesson et al., 2008a). We have shown that the combination of sniff frequency and the recruitment of short-term plasticity create a narrow window of opportunity for phase-locked cortical responses to code changes in stimulus intensity. Such phase-locked codes may be advantageous over mean rate codes when integration times for odor evaluation are short. Another interesting possibility is that increased phase-locked activity across the cortical population enhances spike time correlations or oscillatory activity during sniffing. It is not yet known how cortical ensembles code olfactory information during sniffing, and it is clear that additional studies in awake, behaving animals are required. Nonetheless, this study makes new predictions about how behavioral timescales may interact with synaptic mechanisms to influence cortical coding during sensory processing.

\section{References}

Abbott LF, Regehr WG (2004) Synaptic computation. Nature 431:796-803.

Abbott LF, Varela JA, Sen K, Nelson SB (1997) Synaptic depression and cortical gain control. Science 275:220-224.

Arevian AC, Kapoor V, Urban NN (2008) Activity-dependent gating of lateral inhibition in the mouse olfactory bulb. Nat Neurosci 11:80-87.

Bathellier B, Buhl DL, Accolla R, Carleton A (2008) Dynamic ensemble odor coding in the mammalian olfactory bulb: sensory information at different timescales. Neuron 57:586-598.

Bower JM, Haberly LB (1986) Facilitating and nonfacilitating synapses on pyramidal cells: a correlation between physiology and morphology. Proc Natl Acad Sci U S A 83:1115-1119.

Brody CD, Hopfield JJ (2003) Simple networks for spike-timing-based computation, with application to olfactory processing. Neuron 37:843-852.

Cang J, Isaacson JS (2003) In vivo whole-cell recording of odor-evoked synaptic transmission in the rat olfactory bulb. J Neurosci 23:4108-4116.

Carey RM, Wachowiak M (2011) Effect of sniffing on the temporal structure of mitral/tufted cell output from the olfactory bulb. J Neurosci 31:10615-10626.

Cury KM, Uchida N (2010) Robust odor coding via inhalation-coupled transient activity in the mammalian olfactory bulb. Neuron 68:570-585.

Davison IG, Katz LC (2007) Sparse and selective odor coding by mitral/ tufted neurons in the main olfactory bulb. J Neurosci 27:2091-2101.

Ferrante M, Migliore M, Ascoli GA (2009) Feed-forward inhibition as a buffer of the neuronal input-output relation. Proc Natl Acad Sci U S A 106:18004-18009.

Franks KM, Isaacson JS (2006) Strong single-fiber sensory inputs to olfactory cortex: implications for olfactory coding. Neuron 49:357-363.

Frasnelli J, Charbonneau G, Collignon O, Lepore F (2009) Odor localization and sniffing. Chem Senses 34:139-144.

Fuentes RA, Aguilar MI, Aylwin ML, Maldonado PE (2008) Neuronal activity of mitral-tufted cells in awake rats during passive and active odorant stimulation. J Neurophysiol 100:422-430.

Galán RF, Fourcaud-Trocmé N, Ermentrout GB, Urban NN (2006) Correlation-induced synchronization of oscillations in olfactory bulb neurons. J Neurosci 26:3646-3655.

Giridhar S, Doiron B, Urban NN (2011) Timescale-dependent shaping of correlation by olfactory bulb lateral inhibition. Proc Natl Acad Sci U S A 108:5843-5848.

Hasselmo ME, Bower JM (1990) Afferent and association fiber differences 
in short-term potentiation in piriform (olfactory) cortex of the rat. J Neurophysiol 64:179-190.

Kay LM (2005) Theta oscillations and sensorimotor performance. Proc Natl Acad Sci U S A 102:3863-3868.

Kay LM, Laurent G (1999) Odor- and context-dependent modulation of mitral cell activity in behaving rats. Nat Neurosci 2:1003-1009.

Kepecs A, Uchida N, Mainen ZF (2007) Rapid and precise control of sniffing during olfactory discrimination in rats. J Neurophysiol 98:205-213.

Lei H, Mooney R, Katz LC (2006) Synaptic integration of olfactory information in mouse anterior olfactory nucleus. J Neurosci 26:12023-12032.

Litaudon P, Amat C, Bertrand B, Vigouroux M, Buonviso N (2003) Piriform cortex functional heterogeneity revealed by cellular responses to odours. Eur J Neurosci 17:2457-2461.

Litaudon P, Garcia S, Buonviso N (2008) Strong coupling between pyramidal cell activity and network oscillations in the olfactory cortex. Neuroscience 156:781-787.

Luna VM, Schoppa NE (2008) GABAergic circuits control input-spike coupling in the piriform cortex. J Neurosci 28:8851-8859.

Macrides F, Chorover SL (1972) Olfactory bulb units: activity correlated with inhalation cycles and odor quality. Science 175:84-87.

Mainland J, Sobel N (2006) The sniff is part of the olfactory percept. Chem Senses 31:181-196.

Margrie TW, Schaefer AT (2003) Theta oscillation coupled spike latencies yield computational vigour in a mammalian sensory system. J Physiol 546:363-374.

Markram H, Wang Y, Tsodyks M (1998) Differential signaling via the same axon of neocortical pyramidal neurons. Proc Natl Acad Sci U S A 95:5323-5328.

Mitchell SJ, Silver RA (2003) Shunting inhibition modulates neuronal gain during synaptic excitation. Neuron 38:433-445.

Nagayama S, Enerva A, Fletcher ML, Masurkar AV, Igarashi KM, Mori K, Chen WR (2010) Differential axonal projection of mitral and tufted cells in the mouse main olfactory system. Front Neural Circuits 4:pii:120.

Padmanabhan K, Urban NN (2010) Intrinsic biophysical diversity decorrelates neuronal firing while increasing information content. Nat Neurosci 13:1276-1282.

Poo C, Isaacson JS (2009) Odor representations in olfactory cortex: "sparse" coding, global inhibition, and oscillations. Neuron 62:850-861.

Porter J, Craven B, Khan RM, Chang SJ, Kang I, Judkewitz B, Volpe J, Settles G, Sobel N (2007) Mechanisms of scent-tracking in humans. Nat Neurosci 10:27-29.

Rajan R, Clement JP, Bhalla US (2006) Rats smell in stereo. Science 311:666-670.
Rennaker RL, Chen CF, Ruyle AM, Sloan AM, Wilson DA (2007) Spatial and temporal distribution of odorant-evoked activity in the piriform cortex. J Neurosci 27:1534-1542.

Reyes A, Lujan R, Rozov A, Burnashev N, Somogyi P, Sakmann B (1998) Target-cell-specific facilitation and depression in neocortical circuits. Nat Neurosci 1:279-285.

Rinberg D, Koulakov A, Gelperin A (2006) Sparse odor coding in awake behaving mice. J Neurosci 26:8857-8865.

Shusterman R, Smear MC, Koulakov AA, Rinberg D (2011) Precise olfactory responses tile the sniff cycle. Nat Neurosci 14:1039-1044.

Sobel EC, Tank DW (1993) Timing of odor stimulation does not alter patterning of olfactory bulb unit activity in freely breathing rats. J Neurophysiol 69:1331-1337.

Stokes CC, Isaacson JS (2010) From dendrite to soma: dynamic routing of inhibition by complementary interneuron microcircuits in olfactory cortex. Neuron 67:452-465.

Suzuki N, Bekkers JM (2006) Neural coding by two classes of principal cells in the mouse piriform cortex. J Neurosci 26:11938-11947.

Suzuki N, Bekkers JM (2011) Two layers of synaptic processing by principal neurons in piriform cortex. J Neurosci 31:2156-2166.

Thesen A, Steen JB, Døving KB (1993) Behaviour of dogs during olfactory tracking. J Exp Biol 180:247-251.

Uchida N, Mainen ZF (2003) Speed and accuracy of olfactory discrimination in the rat. Nat Neurosci 6:1224-1229.

Urban NN, Sakmann B (2002) Reciprocal intraglomerular excitation and intra- and interglomerular lateral inhibition between mouse olfactory bulb mitral cells. J Physiol 542:355-367.

Verhagen JV, Wesson DW, Netoff TI, White JA, Wachowiak M (2007) Sniffing controls an adaptive filter of sensory input to the olfactory bulb. Nat Neurosci 10:631-639.

Wesson DW, Carey RM, Verhagen JV, Wachowiak M (2008a) Rapid encoding and perception of novel odors in the rat. PLoS Biol 6:e82.

Wesson DW, Donahou TN, Johnson MO, Wachowiak M (2008b) Sniffing behavior of mice during performance in odor-guided tasks. Chem Senses 33:581-596.

Wesson DW, Verhagen JV, Wachowiak M (2009) Why sniff fast? The relationship between sniff frequency, odor discrimination, and receptor neuron activation in the rat. J Neurophysiol 101:1089-1102.

Youngentob SL, Mozell MM, Sheehe PR, Hornung DE (1987) A quantitative analysis of sniffing strategies in rats performing odor detection tasks. Physiol Behav 41:59-69. 